\title{
New centrality measures for assessing smart grid vulnerabilities and predicting brownouts and blackouts
}

\author{
Pravin Chopade ${ }^{a 1}$, Marwan Bikdash ${ }^{b}$ \\ ${ }^{a}$ Department of Computer Science, College of Engineering, North Carolina A\&ST State \\ University, 526 McNair Building, 1601 East Market Street, Greensboro, North Carolina \\ 27411, USA \\ ${ }^{b}$ Department of Computational Science and Engineering, College of Engineering, North \\ Carolina A $8 T$ State University, 301 Fort IRC, East Market Street, Greensboro, North \\ Carolina 27411, USA
}

\begin{abstract}
This paper proposes mathematical models based on the electrical properties of smart grids for conducting vulnerability analyses and predicting brownouts and blackouts. Definitions of pseudo-Laplacian, pseudo-adjacency and pseudodegree matrices for smart grids are introduced to specify new centrality measures with electrical interpretations. The centrality measures are used to rank the relative importance of nodes (e.g., generating stations or substations) and edges (e.g., transmission lines or buses) of a graph corresponding to a power grid network and to assess the overall vulnerability of the network. The reliability of using the centrality measures to predict brownouts and blackouts is demonstrated in the face of random and targeted attacks.

Monte-Carlo simulations are used to analyze attacks on smart grid networks and to assess the performance of the centrality measures. The simulations employ the IEEE 30-bus, IEEE 57-bus and IEEE 300-bus networks as well as the WSCC 4941-bus real power grid. Every scenario in the MonteCarlo simulations involves the removal of a subset of buses and performing a complete nonlinear Newton-Raphson power flow analysis to compute the power traffic matrix and the corresponding centrality. The Monte-Carlo simulations conclusively demonstrate that electrical centrality measures based on the power traffic matrix are reliable indicators of the total unsatisfied
\end{abstract}

\footnotetext{
${ }^{1}$ Corresponding author:

Pravin Chopade (pvchopad@ncat.edu)

Preprint submitted to IJCIP

(C) 2015. This manuscript version is made available under the Elsevier user license http://www.elsevier.com/open-access/userlicense/1.0/
}

December 3, 2015 
load ratio (i.e., the load taken offline due to an attack divided by the total load demand). A key result is that, if the total centrality score of the removed buses exceeds a threshold estimated via Monte-Carlo simulation, then a sudden and dramatic jump to a blackout situation is ensured.

\section{Keywords}

Smart Grid; Centrality Measures; Admittance; Laplacian Matrix; Vulnerabilities; Robustness

\section{Manuscript No.: IJCIP-D-14-00030}

Submitted: July 21, 2014; Revision 1 Submitted: June 6, 2015; Revision 2 Submitted: October 1, 2015; Accepted: November 25, 2015

\section{Introduction}

In many systems, the removal of certain critical components either accidentally or deliberately can cause system failure. In the case of a power system, a transmission line or generating station failure can seriously impact normal operations, possibly culminating in a brownout or even a blackout. Removing a transmission line shifts the load in the line to other intact lines in order to match the load demand. But this could overload other portions of the network, triggering relays that disconnect additional transmission lines in the power network. This could potentially lead to cascading effects that cause a large portion of the network to suffer total power loss [1,2].

More serious and rapid network loss can occur in the case of node removal. Nodes, especially generating stations, are connected to many other nodes via links such as transmission lines [32]. Large-scale faults can induce failures of several nodes and/or links. Localization indicates that the faulty nodes are located in the same neighborhood [18]. Unlike random graphs, scale-free networks display - on the global and local scales - high degrees of error tolerance as well as extreme vulnerability to attacks. Global and local efficiency may be unaffected by the failure of some randomly chosen nodes, but the networks can be extremely sensitive to the removal of a few nodes that are crucial to maintaining network connectivity [13]. 
Some of the centrality techniques proposed in recent years use concepts from network theory to study various properties of power grids. Centrality indices quantify the fact that some nodes and edges are more central or more important in a network than others [7, 8]. This paper proposes mathematical models that are based on the electrical properties of smart grids for conducting vulnerability analyses and predicting brownouts and blackouts. Definitions of pseudo-Laplacian, pseudo-adjacency and pseudo-degree matrices for smart grids are introduced; this leads to the specification of new centrality measures for which electrical interpretations are developed. The new centrality measures are used to rank the relative importance of nodes and edges of a graph corresponding to a power grid network and to assess the overall vulnerability of the network. Monte-Carlo simulations employing random and targeted attacks on the IEEE 30-bus, IEEE 57-bus and IEEE 300-bus networks and the WSCC 4941-bus real power grid network are used to demonstrate the reliability of using the centrality measures to predict brownout and blackout scenarios. In particular, the simulations conclusively demonstrate that electrical centrality measures based on the power traffic matrix are reliable indicators of the total unsatisfied load ratio (i.e., the load taken offline due to an attack divided by the total load demand). A key result is that, if the total centrality score of the removed buses exceeds a threshold estimated via Monte-Carlo simulation, then a sudden and dramatic jump to a blackout situation occurs.

\section{Related Work}

Network theory has been used to model and analyze several aspects of power system networks. The structural vulnerabilities of the North American power grid have received considerable attention after the North American blackout of August 2003. Large-scale blackouts and cascading failures have also motivated the analysis of the Italian power grid [6].

Bompard et al. [6] have developed an enhanced approach that uses traditional topological metrics (e.g., degrees of nodes and global efficiency) as well as the physical/operational behaviors of a power grid in terms of real power flow allocation on lines and line flow limits. In particular, they specified two new metrics, entropic degree and net-ability, that can be used to assess structural vulnerabilities in power systems. Andersson et al. [4] have summarized important reasons for blackouts such as the lack of reliable real-time data, insufficient time to take decisive and appropriate remedial actions, increased 
failure due to aging equipment and the lack of automated and coordinated controls to take immediate actions in an effort to prevent cascading failures.

Centrality measures the relative importance of a node or link in terms of network efficiency and network resource utilization. Koschutzki et al. [26] examined centrality indices based on distances and neighborhoods as well as on shortest paths. They presented several influential, classical centrality indices based on structural analysis, but they did not strive for completeness or provide a catalog of basic centrality indices along with their applications.

Borgatti [7] states that centrality measures can be regarded as generating expected values for certain kinds of node outcomes (e.g., speed and frequency of reception) given implicit traffic flow models. Borgatti regarded the formulas for centrality concepts such as betweenness and closeness as generating the expected values under specific unstated flow models for certain kinds of node participation in network flows. The principal contribution of Borgatti's work is that it provides assumptions underlying each measure and evaluates each measure using simulation. Node-centric measures are more convenient for computation and interpretation, and are, therefore, more common than edge-centric measures.

Centrality is commonly used in social network analysis to characterize social power and structural influence [28]. However, centrality plays a somewhat different role when studying faults and fault propagation in physical networks such as smart grids [42].

Zio et al. [42] have discussed the limitations that arise when neglecting the actual capacities of links, their failure probabilities and the fact that flows between network nodes are typically a global phenomenon, not restricted to direct or shortest paths as is typically assumed. To overcome some of these limitations, they developed a model of random flow propagation where the notion of betweenness centrality is extended to account for random flows in a network. Zio et al. state that the randomization of the flow out of a node is driven by the capacity values of its outgoing links and allows flows to travel along non-geodesic paths. However, they have not conducted an actual functional power flow analysis.

The notion of an electrical degree centrality measure was introduced by Hines and Blumsack [24]. The electrical equivalent distance between generation nodes and a substation determines its importance. Thus, the importance of a substation is represented by its maximum level of short circuit power. This criterion for determining the importance of a node or substation in a smart grid depends on the number of functional generators (node admit- 
tance).

Several centrality measures have been developed over the years [14, 20, 28], but they are often not customized to study fault patterns or functional vulnerabilities. Most centrality definitions can predict the impact of a node failure when random small failures are considered. However, localized and large-scale faults exhibit properties that are distinct from other types of faults because their impacts are determined by the importance of single nodes as well as the aggregate importance of the entire faulty region. A simpler approach is to aggregate the impact of all the nodes in a region, but this does not take into account the inherent couplings of nodes.

Pinar et al. [33] have studied the power grid vulnerability problem using bi-level mixed integer nonlinear programming (MINLP). They identify small groups of lines whose removal cause severe blackouts. Their analysis reveals a special structure in the formulation that can be exploited to avoid nonlinearity and approximate the original problem as a pure combinatorial problem. The approach leverages the correspondence between the Jacobian matrix (a representation of the feasibility boundary of the equations that describe power flow in a grid) and the Laplacian matrix from spectral graph theory (a graph representation of a power grid) to analyze a reduced combinatorial problem known as the network inhibition problem. A key contribution of Pinar et al. is their presentation of optimization strategies for real-sized power grids. In contrast, the approach presented in this paper relies on node removal based on degree centrality scores. Additionally, four new types of structural and functional centralities are presented. This renders the approach simpler, more efficient and computationally cheaper. The approach also anticipates component outages that could lead to brownouts or blackouts much faster than the method of Pinar et al.

Wang et al. [38] have proposed a two-stage robust optimization approach to solve the N-k-contingency-constrained unit commitment (CCUC) problem. Their approach considers generator and transmission line contingencies and all possible component failure scenarios. They create a two-stage robust optimization problem formulation and develop a decomposition framework to enable tractable computation. Linearization techniques and duality theory are employed to transform the problem into a mixed-integer linear program (MILP). This approach guarantees a globally optimal solution in a finite number of iterations. However, the performance is highly dependent on the selection of the credible contingency set. The approach presented in this paper can produce such sets with minimal computational effort. Additionally, 
network vulnerability identification is much faster than the approach of Wang et al. [38].

Chen et al. [10] have studied the transmission and generation expansion planning problem that involves the design (or augmentation) of a minimal cost electric power system satisfying the N-k- $\varepsilon$ survivability criterion. The survivability criterion, which generalizes the well-known N-k criterion, requires the existence of a feasible power flow satisfying at least $1-\varepsilon$ fraction of the total demand, even with simultaneous failures of up to $\mathrm{k}$ power system components. Chen et al. formulated a mixed-integer nonlinear program (MINLP) to model the problem along with multiple states representing all possible contingency scenarios and the corresponding flow variables to ensure that the $1-\varepsilon$ fraction of the demand can be met. In contrast, the centrality based method for network vulnerability identification presented in this paper is simpler and computationally less expensive. Moreover, Chen et al. only consider transmission and generation expansion planning (not unit commitment) for survivability and they do not consider random or targeted attacks, or other emergency situations, when formulating the notion of survivability. On the other hand, the approach presented in this paper considers all types of situations, including random and targeted attacks, in determining network vulnerabilities. The approach is also more efficient and provides deeper qualitative understanding.

In a subsequent paper, Chen et al. [11] also consider the problem of designing (or augmenting) an electric power system at a minimum cost to satisfy the N-k- $\varepsilon$ survivability criterion. As before, they considered a mixedinteger programming formulation that takes into account transmission and generation expansion. Their algorithm avoids combinatorial explosion in the number of contingencies by seeking vulnerabilities in intermediary solutions and constraining the design space accordingly. Empirical studies involving modified instances of the IEEE 30-bus and IEEE 57-bus networks demonstrate the effectiveness of their approach. They were able to solve the transmission and generation expansion problems in approximately $30 \mathrm{~min}$, while other approaches failed to provide a solution even after two hours. However, Chen et al. [11] do not discuss scalability and performance for networks with more than 57 buses, address issues related to computational complexity or consider any attack situations when formulating the notion of survivability. In contrast, the method proposed in this paper exhibits better performance and is scalable to large networks. Furthermore, the method provides useful statistics that provide deeper insights into network vulnerabilities as well as 
mitigation strategies.

Jin et al. [25] have presented a novel application of parallel betweenness centrality to power grid contingency selection. They cross-validated their method using a model of the Western U.S. power grid implemented on a Cray XMT system. They achieved a speedup of 55 times (on 64 processors) compared with the single-processor version of the same code running on the Cray XMT. They also compared an OpenMP-based version of the same code running on an HP Superdome shared-memory machine. The performance of the Cray XMT code demonstrated better scalability and resource utilization, and shorter execution times for large-scale power grids. The method is expected to provide quick and efficient solutions for massive contingency selection problems to enable power grid operators to identify and mitigate cascading power grid failures in real time. The method of Jin et al. is scalable, but it is not very efficient and is computationally expensive; also, failure situations are not considered, and contingency formulation and analysis are missing. In contrast, the modified degree centrality approach presented in this paper is more efficient, cheaper and dynamic in nature. The approach also considers failures such as brownouts and blackouts under random attacks as well as targeted attacks by knowledgeable adversaries.

Donde et al. [16] have proposed a well-defined optimization problem to help screen for severe multiple contingencies such as disturbances and blackouts. Their screening process is essentially a graph partitioning algorithm. The algorithm finds the minimum change in a network to move the power flow feasibility boundary to the present operating point and cause the system to separate with a user-specified power imbalance. The lines identified by the optimization program are used in a subsequent analysis stage to find combinations that may lead to blackouts. Donde et al. applied their approach to the IEEE 30-bus network. However, their approach does not fully implement spectral graph theory as required by graph partitioning algorithms. Furthermore, the approach does not incorporating a severity function in the optimization problem to avoid trivial solutions or solutions of little consequence. In contrast, the method presented in this paper considers node removal based on different centrality scores; additionally, network vulnerability identification is more efficient and highly scalable.

Electric grids are currently designed for limited resilience to naturallyoccurring incidents such as tornadoes, hurricanes, ice storms and earthquakes. During emergency situations, utility personnel often respond quickly to perform repairs and restore systems that are isolated by protection mech- 
anisms as a result of enduring failures [21]. Given that power systems are already designed for resilience in hostile natural environments, it might appear that cyber attacks would not pose significant new threats. After all, if a cyber attack were to cause problems to an electric power network, protective relays should be able to isolate the problems just as they would in any other emergency situation. Even if protective relays were to be compromised by a cyber attack, power system protective schemes such as remote and local back-up protection can isolate problem areas [40]. However, it is not economically feasible to implement enough redundancy to make power systems $100 \%$ reliable. The redundancy built into power systems enables them to be operated such that the probability of dropping service due to an unanticipated failure is relatively small [15]. If a cyber attacker were to cause random equipment failures, it is reasonable to believe that the cyber attack would pose no more problems than bad weather.

However, intelligent and knowledgeable cyber attackers would not abide by statistical failure probability models. Like electric utility engineers, a cyber attacker with sufficient information could perform a vulnerability analysis of a power system and identify coordinated failures that could cause catastrophic conditions. Therefore, in-depth vulnerability analyses of electric power networks are required to combat coordinated, targeted attacks by malicious actors who are both intelligent and knowledgeable.

Wang et al. [39] have conducted a survey of cyber security in smart grids. They have highlighted smart grid security requirements, network vulnerabilities and attack countermeasures, including secure communications protocols and architectures. Smart grid cyber security is a critical issue because millions of electronic devices will be interconnected via communications networks throughout critical power facilities. Such connectivity in a massive infrastructure has an immediate and potentially massive impact on smart grid reliability.

Viewed in this light, the approach presented in this paper is very useful. The new measures based on degree centrality and the pseudo-adjacency, pseudo-Laplacian and pseudo-degree matrices can be used to determine the structural and functional vulnerabilities of smart grids. Furthermore, the new measures have electrical interpretations that incorporate the connectivity or coupling of nodes; these interpretations are valuable when analyzing vulnerabilities and developing mitigation strategies for smart grids. 


\section{Graph-theoretic matrices and centrality measures}

A network is defined as an object containing elements and interactions (connections) between the elements. A graph $G_{n}(V, E)$ comprising a node set $V$ and link set $E$ is a natural means for modeling networks. Given a graph representation of a network with $N$ nodes and $m$ links or edges, the line-node incidence matrix of the network is an $m \times N$ matrix $M$ where the $l^{\text {th }}$ edge is connected between nodes $i$ and $j$ if and only if:

$$
M:\left\{\begin{array}{l}
M_{l i}=1 \\
M_{l j}=-1 \\
M_{l k}=0 \quad \text { with } k \neq i \text { or } j
\end{array}\right.
$$

The Laplacian matrix $L$ of an $N \times N$ network is defined as [5]:

$$
L=M^{T} M
$$

The elements of the Laplacian matrix $L$ are given by:

$$
L_{i j}:\left\{\begin{array}{cll}
-1 & \text { if a link exists from node } i \text { to node } j & \text { for } j \neq i \\
k & \text { where } k=-\sum_{j \neq i} L_{i j} & \text { for } j=i \\
0 & \text { otherwise } &
\end{array}\right.
$$

where $i, j=1,2, \cdots, N$. The matrix $L$ is positive semidefinite, real symmetric and the elements of every row (and column) sum to zero [31]. Alternatively:

$$
L=D-A
$$

where $D=\operatorname{diag}(L)$ is the diagonal degree matrix of the network and $A$ is the $N \times N$ adjacency matrix. The adjacency matrix can be written as [27]:

$$
A_{i j}: \begin{cases}1 & \text { if an edge exists from node } i \text { to node } j \\ 0 & \text { if no edge exists from node } i \text { to node } j\end{cases}
$$

Note that $A_{i j}=k$ if there are $k$ parallel edges from $i$ to $j$. Moreover, $D_{i} \geq 0$ and $D_{i i}$ is the number of edges connected to node $i$. Note that the diagonal elements of the Laplacian matrix are assumed to be positive.

Centrality indices are quantifications of the fact that some nodes and/or edges in a network are more central or more important than others. Different centrality indices are suitable for different applications, but most indices have 
structural significance and require that a network be connected [26]. Centrality indices that involve a volume or length of a walk are usually referred to as radial (distance and neighborhood) indices.

The simplest centrality measure is degree centrality. The degree centrality of a node is its node degree or number of neighbors (i.e., number of edges incident on the node). This centrality score represents the connectivity of the node to the rest of the network and reflects the immediate ability of the node to exert its influence on the rest of the network or to be exposed to whatever flows in the network (e.g., disturbances, shared information, power or traffic flows, even a virus) $[8,19]$. For a graph $G_{n}=(V, E)$ with $N$ nodes, where $V$ is the set of nodes and $E$ is the set of edges, given its Laplacian $L$, the degree centrality $C_{i}^{D}$ of a node $i$ is computed as $[8,19]$ :

$$
C_{i}^{D}=\frac{D_{i}}{2 n_{E}}=\frac{L_{i i}}{2 n_{E}}
$$

where $2 n_{E}=\operatorname{Tr}(D)=\operatorname{Tr}(L)$ is used as a normalization factor so that $0 \leq$ $C_{i}^{D} \leq 1$ and $\sum_{i} C_{i}^{D}=1$. Note that $n_{E}$ is the total number of edges in the graph. Also, the degree of node $i$ is computed as the sum of the corresponding row (or column) in the adjacency matrix. Degree centrality is clearly a local measure and does not necessarily indicate the importance of a node in the overall graph. Another local centrality measure is the clustering coefficient.

\section{New electrical centrality measures}

This section describes new electrical centrality measures for assessing the structural and functional vulnerabilities of smart grid networks [12]. The new centrality measures are derived from the electrical and structural properties of smart grid networks. Two standard centrality measures are applied to graph and weighted graph representations of a smart grid network. These measures are used to rank the relative importance of nodes and edges in a network and to assess the overall vulnerability of the network.

New pseudo-Laplacian, pseudo-adjacency and pseudo-degree matrices are introduced to analyze the performance of smart grid networks [12]. Using these weighted graph matrices, four new centrality measures based on degree centrality are introduced. These centrality measures consider the importance of electrical couplings in a power network using admittance and power flow. The new centrality measures are compared and their electrical interpretations are discussed. 


\subsection{Functional dynamics of power flow}

A smart grid network comprises several buses that are interconnected by transmission lines. Power is injected into a bus from connected generators and loads are tapped from the bus. There are always some buses with no generation facilities; also, there may be others with (voltage-ampere reactive) generators attached. The surplus power at some buses is transported via transmission lines to buses that are deficient in power. Typically, a transmission line is expressed using a nominal- $\pi$ (or equivalent- $\pi$ for long lines) model while line resistance is almost always neglected in load flow analysis.

The dynamics of a power network are designated by the network admittance matrix $Y$ and by the power generation, distribution and load settings. The power network dynamics are governed by the network equation [22, 37]:

$$
I=Y_{\text {bus }} V=\left(Y+Y^{L}\right) V
$$

where $V$ is the complex bus voltage vector, $I$ is the injected current vector and $Y_{\text {bus }}$ is the $N \times N$ network or bus admittance matrix that is determined by the connecting topology and its electrical parameters. $Y$ is the part of the bus admittance matrix that excludes the admittance of the bus to ground. It is given by:

$$
Y^{L}=\operatorname{diag}\left(y_{1}^{L}, y_{2}^{L}, \cdots, y_{N}^{L}\right)
$$

The off-diagonal elements of $Y$ are equal to the negative of the admittance values between the nodes. These are referred to as the mutual admittance or transfer admittance:

$$
Y=G+j B \in \mathbb{R}^{N \times N} \text { where } \begin{cases}Y_{i i}=\sum_{j=1}^{N} y_{i j} & \text { for } j=i \\ Y_{i j}=Y_{j i}=-y_{i j} & \text { for } j \neq i\end{cases}
$$

Note that $y_{i j}=\frac{1}{z_{i j}}$ where $z_{i j}=r_{i j}+j x_{i j}$ is the series impedance of the transmission line connecting buses $i$ and $j$. The following general notation is used:

$g_{i j}=\operatorname{Re}\left\{y_{i j}\right\}, b_{i j}=\operatorname{Im}\left\{y_{i j}\right\}, G_{i j}=\operatorname{Re}\left\{Y_{i j}\right\}, r_{i j}=\operatorname{Re}\left\{z_{i j}\right\}, x_{i j}=\operatorname{Im}\left\{z_{i j}\right\}$

The admittance matrix relates current injections at a bus to the bus voltages. It is a fundamental network analysis tool that is heavily used in this work. $Y_{i j}$ is the admittance of the line connecting buses $i$ and $j$. 
Figure 1: Equivalent $\pi$ model of a transmission line.

A transmission line is modeled using an equivalent- $\pi$ network as shown in Figure 1. The term $z_{i j}$ denotes the series impedance and the shunt impedances contribute the terms $1 / z_{i j}^{\text {shunt }}$ to $y_{i}^{L}$ and $y_{j}^{L}$. Note that $y_{i j}=0$ when no connection exists between buses $i$ and $j$. Also, note that $y_{i i}=0$ and the admittance from bus $i$ to ground is accounted for in $y_{i}^{L}$. Although $Y_{b u s}$ is sparse, its inverse $Z_{b u s}=Y_{b u s}^{-1}$ is not sparse. Hence, it is preferable to work with $Y_{\text {bus }}$ and to separate it into its components $Y$ and $Y^{L}$.

Therefore, from Equation (9):

$$
B_{i j}=\operatorname{Im} Y_{i j}=\left\{\begin{array}{cc}
\sum_{j=1}^{N} b_{i j} & \text { for } j=i \\
-b_{i j} & \text { for } j \neq i
\end{array}\right.
$$

The smart grid network is modeled using the polar-coordinate formulation of the standard power flow equations, which are solved using NewtonRaphson load flow analysis [37]. At a given node, the bus power injections can be due to synchronous generators or renewable sources such as wind or solar power. Loads are modeled as active or reactive power withdrawals while passive loads are modeled as elements of $Y^{L}$. The formulation is general enough to account for the possibility of loads at every bus in the network [9].

In load flow and power flow analyses, each bus of a power system network can be associated with four variables: (i) voltage magnitude $\left|V_{i}\right|$; (ii) voltage phase angle $\theta_{i}$; (iii) net real power injected $P_{i}$; and (iv) net reactive power injected $Q_{i}$.

The complex power injected by the source into the bus $i$ is given by [23]:

$$
S_{i}=P_{i}+j Q_{i}=V_{i} I_{i}^{*}, i=1,2, \ldots, N
$$

However, from Equation (7):

$$
I_{i}=\sum_{j=1}^{N}\left(Y_{i j}+Y_{i j}^{L}\right) V_{j}=\sum_{j=1}^{N} Y_{i j} V_{j}+y_{i}^{L} V_{i}
$$

because $\sum_{j=1}^{N} Y_{i j}^{L} V_{j}=Y_{i i}^{L} V_{i}=y_{i}^{L} V_{i}$. Taking the complex conjugate of Equa- 
Figure 2: Smart grid bus network.

tion (13) and substituting the value of $I_{i}^{*}$ in Equation (12) yields:

$$
S_{i}=V_{i}\left(\sum_{j=1}^{N} Y_{i j} V_{j}\right)^{*}+y_{i}^{*} V_{i} V_{i}^{*}, i=1,2, \ldots, N
$$

where $V_{i}=\left|V_{i}\right| \angle \theta_{i}$ and $Y_{i j}=G_{i j}+j B_{i j}$. Then, Equation (14) may be written as:

$$
S_{i}=\sum_{j=1}^{N}\left(\left|V_{i}\right|\left|V_{j}\right| \angle\left(\theta_{i}-\theta_{j}\right)\right)\left(G_{i j}-j B_{i j}\right)+y_{i}^{*}\left|V_{i}\right|^{2}
$$

Using the Euler relation, a phasor can be expressed as a complex function of sinusoids:

$$
V=|V| \angle \theta=|V|(\cos \theta+j \sin \theta)
$$

Equation (15) then becomes:

$$
S_{i}=\sum_{j=1}^{N}\left(\left|V_{i}\right|\left|V_{j}\right| \cos \left(\theta_{i}-\theta_{j}\right)+j \sin \left(\theta_{i}-\theta_{j}\right)\right)\left(G_{i j}-j B_{i j}\right)+\left(y_{i}^{L}\right)^{*}\left|V_{i}\right|^{2}
$$

Algebraically multiplying the two terms inside the parentheses in Equation (17) and separating the real and imaginary parts (recall that $S_{i}=$ $P_{i}+j Q_{i}$ and $\left.y_{i}^{L}=g_{i}^{L}+j b_{i}^{L} \Rightarrow\left(y_{i}^{L}\right)^{*}=g_{i}^{L}-j b_{i}^{L}\right)$ enables the expression of Equation (17) in terms of two equations, one for the real part $P_{i}$ and the other for the imaginary part $Q_{i}$ :

$$
\begin{aligned}
P_{i} & =\sum_{j=1}^{N}\left|V_{i}\right|\left|V_{j}\right|\left(G_{i j} \cos \left(\theta_{i}-\theta_{j}\right)+B_{i j} \sin \left(\theta_{i}-\theta_{j}\right)\right)+G_{i}^{L}\left|V_{i}\right|^{2} \\
Q_{i} & =\sum_{j=1}^{N}\left|V_{i}\right|\left|V_{j}\right|\left(G_{i j} \sin \left(\theta_{i}-\theta_{j}\right)-B_{i j} \cos \left(\theta_{i}-\theta_{j}\right)\right)-B_{i}^{L}\left|V_{i}\right|^{2}
\end{aligned}
$$

Figure 2 shows a smart grid bus network. For bus $i$, let $V_{i}$ denote the bus voltage magnitude and $\theta_{i}$ denote the bus voltage angle. Let $P_{i}^{G}$ and $Q_{i}^{G}$ denote the active and reactive power injections from the $i^{\text {th }}$ synchronous generator and $P_{i}^{W}$ denote active power injections from the $i^{\text {th }}$ wind or solar 
power plant. Finally, let $P_{i}^{D}$ and $Q_{i}^{D}$ be the active and reactive power demands, respectively. Note that it is convenient to consider loads as negative generators and lump together the generation and load powers at the buses.

The injected real power and reactive power at bus $i$ are given by [3, 9, 22]:

$$
\begin{aligned}
P_{i} & =P_{i}^{G}+P_{i}^{W}-P_{i}^{D} \\
Q_{i} & =Q_{i}^{G}+Q_{i}^{W}-Q_{i}^{D}
\end{aligned}
$$

Then,

$$
\begin{aligned}
& P_{i}^{F}=P_{i}-\left|V_{i}\right|^{2} G_{i}^{L}=P_{i}^{G}+P_{i}^{W}-P_{i}^{D}-\left|V_{i}\right|^{2} G_{i}^{L} \\
& Q_{i}^{F}=Q_{i}+\left|V_{i}\right|^{2} B_{i}^{L}=Q_{i}^{G}+Q_{i}^{W}-Q_{i}^{D}+\left|V_{i}\right|^{2} B_{i}^{L}
\end{aligned}
$$

where $P_{i}^{F}$ is the total real power leaving the bus over the network.

Depending on the problem of interest, two of the following four variables are known for each bus in a network: $P_{i}, Q_{i},\left|V_{i}\right|$ and $\theta_{i}$. Thus, for each bus, there are two equations available for $Q_{i}$ and $\theta_{i}$ given $P_{i}$ and $\left|V_{i}\right|$ and two unknown variables. For power flow problems, it is assumed that $P_{i}$ and $\left|V_{i}\right|$ are known and that it is necessary to solve for $Q_{i}$ and $\theta_{i}$. If no solution exists, then a blackout is expected, unless some islanding is imposed. After the voltage magnitudes and angles for each bus are known, the slack bus power injections and line flows can be calculated. For the line flows, the following equations are employed:

$$
\begin{aligned}
P_{i}^{F} & =\sum_{j=1}^{N}\left|V_{i}\right|\left|V_{j}\right|\left(G_{i j} \cos \left(\theta_{i}-\theta_{j}\right)+B_{i j} \sin \left(\theta_{i}-\theta_{j}\right)\right) \\
Q_{i}^{F} & =\sum_{j=1}^{N}\left|V_{i}\right|\left|V_{j}\right|\left(G_{i j} \sin \left(\theta_{i}-\theta_{j}\right)-B_{i j} \cos \left(\theta_{i}-\theta_{j}\right)\right)
\end{aligned}
$$

Note that $P_{i}^{F}$ can be written as $P_{i}^{F}=\sum_{j=1}^{N} P_{i j}^{F}$ where

$$
P_{i j}^{F}=\left|V_{i}\right|\left|V_{j}\right|\left(G_{i j} \cos \left(\theta_{i}-\theta_{j}\right)+B_{i j} \sin \left(\theta_{i}-\theta_{j}\right)\right)
$$

and hence,

$$
P_{i j}^{F}+P_{j i}^{F}=2\left|V_{i}\right|\left|V_{j}\right| G_{i j} \cos \left(\theta_{i}-\theta_{j}\right)>0
$$


which represents the total real power loss due to transmission on the line connecting buses $i$ and $j$. If $i=j$, the following equation is obtained:

$$
P_{i i}^{F}=\left|V_{i}\right|^{2} G_{i i} \cos \left(\theta_{i}-\theta_{j}\right)=0
$$

under the assumption that the conductance from the bus to ground is accounted for separately in $y_{i}^{L}$ and, hence, $G_{i i}=0$.

\subsection{Approximate power flow equations (ideal case)}

In this ideal case, the series resistance of the transmission line is relatively small, the angle differences between the connected buses are small and all the bus voltages are approximately unity per-unit. In other words, the following assumption holds:

Assumption 1. For a transmission line connecting buses $i$ and $j$ whose series impedance is given by $r_{i j}+j x_{i j}$ :

1. $r \ll x$, i.e., the resistance of transmission circuits is significantly less than the reactance.

2. $\left|\theta_{i}-\theta_{j}\right| \leq 15^{\circ}$ or 0.262 radians.

3. $0.95<\left|V_{i}\right|$ and $\left|V_{j}\right|<1.05$ in per-units.

The implications of Assumption 1.1 are: if $r \ll x$, then (upon dropping subscripts):

$$
y=\frac{1}{z}=\frac{1}{r+j x}=\frac{r}{r^{2}+x^{2}}+j \frac{-x}{r^{2}+x^{2}}=g+j b
$$

where

$$
g=\frac{r}{r^{2}+x^{2}}>0 \text { and } b=\frac{-x}{r^{2}+x^{2}}<0 \text { typically }
$$

Moreover,

$$
g=\frac{1}{r+\frac{x^{2}}{r}} \approx \frac{r}{x^{2}} \ll 1
$$

In short,

$$
b_{i j} \approx-\frac{1}{x_{i j}}<0 \text { and } g_{i j} \approx \frac{r_{i j}}{x_{i j}^{2}} \ll 1 \text { and }\left|y_{i j}\right| \approx\left|b_{i j}\right|
$$


Therefore, Equations (20a and 20b) can be approximated as:

$$
\begin{aligned}
& P_{i}^{F}=\sum_{j=1}^{N}\left|V_{i}\right|\left|V_{j}\right|\left(B_{i j} \sin \left(\theta_{i}-\theta_{j}\right)\right) \\
& Q_{i}^{F}=\sum_{j=1}^{N}\left|V_{i}\right|\left|V_{j}\right|\left(-B_{i j} \cos \left(\theta_{i}-\theta_{j}\right)\right)
\end{aligned}
$$

The small angle difference in Assumption 1.2 implies $\left|\sin \left(\theta_{i}-\theta_{j}\right)\right| \leq 0.26$, and:

$$
1-\left|\cos \left(\theta_{i}-\theta_{j}\right)\right| \leq 0.034 \text { and } \sin \left(\theta_{i}-\theta_{j}\right) \cong \theta_{i}-\theta_{j} \text { (in radians) }
$$

It is extremely rare to see an angular separation exceed 30 degrees. Thus, it is appropriate to say that the angular separation across any transmission circuit is "small." As the angle $\delta=\theta_{i}-\theta_{j}$ becomes smaller, the cosine function approaches one. Applying these conclusions and Assumption 1.2 to Equations (29a and 29b) yields:

$$
\begin{aligned}
P_{i}^{F} & =\sum_{j=1}^{N}\left|V_{i}\right|\left|V_{j}\right|\left(B_{i j}\left(\theta_{i}-\theta_{j}\right)\right) \\
Q_{i}^{F} & =\sum_{j=1}^{N}\left|V_{i}\right|\left|V_{j}\right|\left(-B_{i j}\right)
\end{aligned}
$$

The following equation is obtained from Assumption 1.3:

$$
P_{i}^{F} \cong \sum_{j=1}^{N} B_{i j}\left(\theta_{i}-\theta_{j}\right)
$$

Using $\left|V_{i}\right| \simeq 1$ and $G_{i j} \approx 0$ :

$$
Q_{i}^{F} \cong-\sum_{j=1}^{N} B_{i j} \cos \left(\theta_{i}-\theta_{j}\right)
$$

The statements about power flow can be narrowed as follows:

$$
\sum_{j \neq i}^{N} B_{i j}=\sum_{k \neq i}^{N} B_{i k}=-B_{i i}
$$


Therefore,

$$
P_{i}^{F}=-\theta_{i} B_{i i}-\sum_{j \neq i}^{N} B_{i j} \theta_{j}=-\sum_{j=1}^{N} B_{i j} \theta_{j}
$$

Or, in matrix notation:

$$
P^{F} \cong-B \theta
$$

where $P^{F} \in \mathbb{R}^{N}, \theta \in \mathbb{R}^{N}$.

Thus, Equation (33) can be written as:

$$
P_{i}^{F}=\sum_{j=1, j \neq i}^{N} B_{i j}\left(\theta_{i}-\theta_{j}\right)
$$

If $P^{F}$ is known, as is usually assumed for power flow calculations, then the approximate angles can be obtained by solving Equation (36) to obtain:

$$
\theta \cong-B^{-1} P^{F}
$$

\subsection{Perturbation of power flow equations around an operating point (quasi-} ideal case)

In this quasi-ideal case, larger perturbations or deviations from the ideal case are assumed.

Assumption 2. The perturbations $\Delta V_{i}, \Delta \theta_{i}, \Delta P_{i}$ and $\Delta Q_{i}$, of the corresponding quantities are relatively small:

$$
\begin{gathered}
\left|G_{i j} \sin \left(\theta_{i}-\theta_{j}\right)\right| \ll\left|B_{i j} \cos \left(\theta_{i}-\theta_{j}\right)\right| \\
\Delta V_{i} \ll V_{i}, \Delta \theta_{i} \ll \theta_{i}, \Delta P_{i} \ll P_{i}, \text { and } \Delta Q_{i} \ll Q_{i}
\end{gathered}
$$

From Equation (20a), for real power flow:

$$
P_{i}+\Delta P_{i}=\sum\left|V_{i}+\Delta V_{i}\right|\left|V_{j}+\Delta V_{j}\right|\left(G_{i j} \cos \left(\theta_{i}+\Delta \theta_{i}-\theta_{j}-\Delta \theta_{j}\right)+B_{i j} \sin \left(\theta_{i}-\theta_{j}+\Delta \theta_{i}-\Delta \theta_{j}\right)\right)
$$

Assume that $\left|V_{i}+\Delta V_{i}\right|\left|V_{j}+\Delta V_{j}\right| \approx\left|V_{i}\right|\left|V_{j}\right|$, then:

$$
P_{i}+\Delta P_{i}=\sum\left|V_{i}\right|\left|V_{j}\right|\left(G_{i j} \cos \left(\theta_{i}-\theta_{j}+\Delta \theta_{i}-\Delta \theta_{j}\right)+B_{i j} \sin \left(\theta_{i}-\theta_{j}+\Delta \theta_{i}-\Delta \theta_{j}\right)\right)
$$


Using a Taylor series expansion, the following equation is obtained:

$$
\Delta P_{i}=\sum_{\substack{j=1 \\ j \neq i}}^{N}\left[-G_{i j} \sin \left(\theta_{i}-\theta_{j}\right)+B_{i j} \cos \left(\theta_{i}-\theta_{j}\right)\right]\left(\Delta \theta_{i}-\Delta \theta_{j}\right)
$$

According to Equation (40), the $G_{i j}$ term may be omitted to yield:

$$
\Delta P_{i}=\sum_{j=1, j \neq i}^{N}\left|V_{i}\right|\left|V_{j}\right|\left(B_{i j} \cos \left(\theta_{i}-\theta_{j}\right)\right)\left(\Delta \theta_{i}-\Delta \theta_{j}\right)
$$

Similar reasoning can be applied to the reactive power to yield:

$$
\begin{aligned}
Q_{i}+\Delta Q_{i} & =\sum_{j=1}^{N}\left|V_{i}+\Delta V_{i}\right|\left|V_{j}+\Delta V_{j}\right| G_{i j} \sin \left(\theta_{i}+\Delta \theta_{i}-\theta_{j}-\Delta \theta_{j}\right) \ldots \\
& \left.-B_{i j} \cos \left(\theta_{i}+\Delta \theta_{i}-\theta_{j}-\Delta \theta_{j}\right)\right)
\end{aligned}
$$

and hence,

$$
\Delta Q_{i}=\sum_{j=1}^{N}\left|V_{i}\right|\left|V_{j}\right|\left[G_{i j} \cos \left(\theta_{i}-\theta_{j}\right)+B_{i j} \sin \left(\theta_{i}-\theta_{j}\right)\right]\left(\Delta \theta_{i}-\Delta \theta_{j}\right)
$$

Further simplication using Assumption 1.3 gives:

$$
\begin{aligned}
\Delta P_{i} & =\sum_{j=1, j \neq i}^{N}\left(B_{i j} \cos \left(\theta_{i}-\theta_{j}\right)\right)\left(\Delta \theta_{i}-\Delta \theta_{j}\right) \\
\Delta Q_{i} & =\sum_{j=1}^{N} G_{i j} \cos \left(\theta_{i}-\theta_{j}\right)\left(\Delta \theta_{i}-\Delta \theta_{j}\right)+\sum_{j=1}^{N} B_{i j} \sin \left(\theta_{i}-\theta_{j}\right)\left(\Delta \theta_{i}-\Delta \theta_{j}\right)
\end{aligned}
$$

\subsection{Describing vulnerabilities using weighted graphs}

For a purely topological representation of a simple graph (with no parallel or self loops), the graph-theoretic matrices satisfy the following properties:

- The adjacency matrix $A$ is real symmetric with zeroes along the diagonal; the other entries are either 0 or 1 . Since the trace is zero, some of the eigenvalues must be positive and others must be negative; hence, the matrix is not sign definite. The adjacency matrix is obtained from the susceptance matrix by zeroing its diagonal elements. 
- The Laplacian matrix $L$ is real symmetric and the sum of each row is zero. The diagonal elements are nonegative and the off-diagonal elements are non-positive, either 0 or -1 .

- The degree matrix $D$ is diagonal with diagonal elements either 0 or 1 .

If parallel links are allowed between nodes, then non-zero entries can have integer values greater than 1 , but with the same sign. If self loops are allowed, the adjacency matrix can have non-negative integer diagonal elements. In any case, the matrices are related by $L=D-A$.

Eliminating the restriction that matrix elements be binary values or integers, leads to the definitions of pseudo-adjacency, pseudo-Laplacian and pseudo-degree matrices. The guidelines discussed above are used to define the pseudo-adjacency, pseudo-Laplacian and pseudo-degree matrices from the power flow equations. These matrices are required to maintain the basic structures and properties of their graph-theoretic counterparts. In particular:

- The pseudo-adjacency matrix $\tilde{A}$ is real symmetric with zeroes along the diagonal; the other entries are non-negative. Since the trace is zero, some of the eigenvalues must be positive and others must be negative; hence, the matrix is not sign definite.

- The pseudo-Laplacian matrix $\tilde{L}$ is real symmetric and the sum of each row is zero. The diagonal elements are non-negative and the off-diagonal elements are non-positive.

- The pseudo-degree matrix $\tilde{D}$ is diagonal with non-negative diagonal elements. The sum of the diagonal elements is twice the total susceptance of all the lines in the system.

Similarly, it is required that $\tilde{L}=\tilde{D}-\tilde{A}$. Note, however, that the entries need not be integers or $-1,1$ or 0 .

\subsubsection{Susceptance matrix}

The power flow problem is to solve the power flow equations (Equations (20a and 20b)) for the remaining two variables per bus. The power flow equations are nonlinear and it is prohibitively expensive to solve them for all possible faults to determine the vulnerabilities. Therefore, it is necessary to develop methods that can deduce or predict the most vulnerable nodes or subnetworks without having to exhaustively simulate all possible 
faults. According to Kirchhoff's law, current and power follow the path of least resistance or, alternatively, admittance is a measure of the ease with which power flows through a transmission line [22].

The real power flow across circuits is determined by the difference in voltage phasor angles between the terminating buses. Note that the susceptance matrix $B$ defined in Equation (11) is real symmetric with negative off-diagonal entries, and every row sums to zero. Therefore $B$, the imaginary part of the admittance matrix, can be interpreted as a pseudo-Laplacian matrix, which is denoted as $\tilde{L}^{B}$ :

$$
B=\tilde{L}^{B}= \begin{cases}\sum_{j=1}^{N} b_{i j}>0 \text { with } b_{i i}=0 & \text { for } j=i \\ b_{i j}=-\frac{x_{i j}}{r_{i j}^{2}+x_{i j}^{2}}<0 & \text { for } j \neq i\end{cases}
$$

This matrix can be further decomposed into a diagonal part called the pseudo-degree matrix $\tilde{D}^{B}$ and a zero-diagonal matrix called the pseudoadjacency matrix $\tilde{A}^{B}$. In other words: $\tilde{L}^{B}=\tilde{D}^{B}-\tilde{A}^{B}$. Let $\tilde{D}^{B}$ be the diagonal part of $B=\tilde{L}^{B}$ that has positive diagonal elements (i.e., $\tilde{D}^{B}=\tilde{L}_{i i}^{B}$ ). Here, $\tilde{D}^{B}$ is the pseudo-diagonal degree matrix, which is obtained by taking the diagonal of the pseudo-Laplacian matrix. Thus:

$$
\begin{aligned}
\tilde{D}_{i i}^{B} & =\text { Sum of all susceptances connected to bus } i \text { (diagonal elements) } \\
& =\sum_{j=1}^{N} b_{i j}
\end{aligned}
$$

Moreover, $\tilde{A}^{B}$ is interpreted as the pseudo-adjacency matrix or B-adjacency matrix:

$$
\begin{aligned}
\tilde{A}_{i j}^{B} & =\text { Susceptance of lines connecting buses } i \text { and } j \\
& =\left\{\begin{array}{cl}
b_{i i}=0 & \text { for diagonal elements }(j=i) \\
b_{i j}=\frac{x_{i j}}{r_{i j}^{2}+x_{i j}^{2}} & \text { for non-diagonal elements }(j \neq i)
\end{array}\right.
\end{aligned}
$$

Note that a unit of susceptance can be viewed as one edge and two units of susceptance can be viewed as a parallel combination of two edges.

\subsubsection{Modified susceptance matrix}

Equation (48) defines the matrix $B_{i j} \cos \left(\theta_{i}-\theta_{j}\right)$. This matrix $B^{\cos }$ (= $\left.B_{i j} \cos \left(\theta_{i}-\theta_{j}\right)\right)$ is the new pseudo-Laplacian (modified susceptance matrix) 
denoted by $\tilde{L}^{B^{\text {cos }}}$; it depends on the term $\cos \left(\theta_{i}-\theta_{j}\right)$. This is similar to Equation (38), but it is obtained under less stringent conditions. Instead of Equation (30), Equation (40) is used to obtain:

$$
\tilde{L}_{i j}^{B^{\cos }}=B_{i j} \cos \left(\theta_{i}-\theta_{j}\right) \quad \text { and } \quad \tilde{L}_{i i}^{B^{\cos }}=\tilde{L}_{i i}^{B}=B_{i i}
$$

The corresponding pseudo-degree matrix $\tilde{D}^{B^{\cos }}$ has the diagonal elements of matrix $\tilde{L}^{B^{\text {cos }} \text { : }}$

$$
\tilde{D}_{i i}^{B^{\cos }}=\sum_{j} B_{i j} \cos \left(\theta_{i}-\theta_{j}\right)
$$

The corresponding pseudo-adjacency $\tilde{A}^{B^{\text {cos }}}$ matrix is:

$$
\begin{aligned}
\tilde{A}_{i i}^{B^{\cos }} & =\tilde{D}_{i i}^{B^{\cos }}-\tilde{L}_{i j}^{B^{\cos }} \\
& = \begin{cases}b_{i i}=0 & \text { for diagonal elements }(j=i) \\
b_{i j} \cos \left(\theta_{i}-\theta_{j}\right) & \text { for non-diagonal elements }(j \neq i)\end{cases}
\end{aligned}
$$

\subsubsection{Power traffic matrix}

The power flowing through a power network is dependent on the transmission line parameters and node voltages [34]. To simplify the treatment, a line is assumed to be lossless, which means that the resistance is negligible. The power flow is thus dependent on the node voltages and line reactances. In this case, the active power transmitted from node $i$ to node $j$ in the power network can be derived in a similar manner as Equation (38).

The general power flow adjacency matrix is given by:

$$
\tilde{A}_{i j}^{P T} B= \begin{cases}\left|P_{i j}^{F}\right| & \text { for } j \neq i \\ 0 & \text { for } j=i\end{cases}
$$

which is typically almost symmetric. The lack of symmetry results from the power loss on the transmission lines. Note, however, that in the ideal case, from Equation (22):

$$
\left|P_{i j}^{F}\right| \approx\left|B_{i j}\right|\left|\theta_{i}-\theta_{j}\right|=\left|P_{j i}^{F}\right|
$$

which makes the matrix symmetric. The matrix $\left|P_{i j}^{F}\right|$ under Assumption 1 is referred to as the power traffic matrix $P T$, which is a real symmetric matrix with zeroes along the diagonal and non-negative off-diagonal elements.

The corresponding power traffic pseudo-degree matrix $\tilde{D}^{P T}$ has diagonal elements:

$$
\tilde{D}_{i i}^{P T}=\sum_{k \neq i}\left|P_{i k}^{F}\right|
$$


Clearly, this indicates the importance of bus $i$ in handling power traffic in the network. It ignores the power generation/consumption at bus $i$. The corresponding pseudo-Laplacian matrix is given by:

$$
\tilde{L}^{P T}=\tilde{D}^{P T}-\tilde{A}^{P T}
$$

Note that these definitions are acceptable in general, including for Assumption 1 (ideal case) and Assumption 2 (perturbation case).

\subsubsection{Power loss matrix}

The power loss adjacency matrix $\tilde{A}_{i j}^{W}$ gives the real power loss between bus $i$ and bus $j$ :

$$
W_{i j}=\tilde{A}_{i j}^{W}=\frac{1}{2}\left(P_{i j}^{F}+P_{j i}^{F}\right)>0
$$

where $P_{i j}^{F}$ is the real power flow between bus $i$ and bus $j$ (see Equation (21a)), which is calculated using Newton-Raphson load flow analysis.

The corresponding pseudo-degree matrix $\tilde{D}_{i j}^{W}$ is a diagonal matrix with elements:

$$
\tilde{D}_{i i}^{W}=\sum_{j} \tilde{A}_{i j}^{W}=\sum_{j}\left(\frac{1}{2}\left(P_{i j}^{F}+P_{j i}^{F}\right)\right)
$$

This matrix represents the total power traffic going through bus $i$ (over transmission lines and not including the power dissipated at the bus). The corresponding pseudo-Laplacian matrix is given by:

$$
\tilde{L}_{i j}^{W}=\tilde{D}_{i i}^{W}-\tilde{A}_{i j}^{W}
$$

\section{Electrical interpretations of degree based functional centralities}

This section describes the electrical interpretations of the new degree based functional centralities [12].

\subsection{Susceptance based degree centrality}

This notion of centrality, which is based on the pseudo-Laplacian matrix taking the place of the susceptance matrix, is also referred to as electrical admittance based centrality or susceptance based degree centrality. The connectivity or coupling of a node with the rest of a smart grid network is related to the number of links it connects as well as the connection strength of each link. Since the admittance of each link reflects the coupling strength, 
the pseudo-Laplacian matrix $\tilde{L}^{B}$ can be used to estimate the centrality of different nodes/buses from a functional (electrical) point of view. Recall that from Equation (28), $\left|y_{i j}\right| \approx\left|b_{i j}\right|$ holds for most transmission lines and, hence, the matrix $\tilde{L}^{B}$ is the dominant part of the admittance matrix. Thus, using Equation (28), the electrical admittance based centrality $C_{i}^{D}(B)$ can be defined as:

$$
C_{i}^{D}(B)=\frac{\tilde{L}_{i i}^{B}}{\sum \tilde{L}_{i i}^{B}}=\frac{\sum_{k} b_{i k}}{\sum_{i} \sum_{k} b_{i k}}
$$

This centrality score can be given the following electrical interpretation:

$$
\begin{aligned}
C_{i}^{D}(B) & =\frac{\text { Total susceptance connected to bus } i}{\text { Total susceptance of the network }} \\
& =\frac{\text { Net reactive power flow (ideal case) }}{\text { Total net reactive power flow in the network (ideal case) }}
\end{aligned}
$$

where $\tilde{L}_{i i}^{B}$ is the diagonal susceptance matrix and $\sum \tilde{L}_{i i}^{B}$ is a normalization factor.

\subsection{Modified susceptance based degree centrality}

This notion of centrality is based on the modified pseudo-Laplacian ma$\operatorname{trix} \tilde{L}^{B^{\text {cos }}}$ (quasi-ideal case) given by Equation (53), i.e., $\tilde{L}_{i j}^{B_{\text {cos }}}=B_{i j} \cos \left(\theta_{i}-\theta_{j}\right)$. In this case, the power flow is given by Equation (48).

Thus, using the matrix $\tilde{L}^{B^{\cos }}$ leads to the modified susceptance based degree centrality $C_{i}^{D}\left(B^{\cos }\right)$ :

$$
\begin{aligned}
C_{i}^{D}\left(B^{\cos }\right) & =\frac{\tilde{D}_{i i}^{B^{\cos }}}{\sum_{i} \tilde{D}_{i i}^{B^{\cos }}}=\frac{B_{i i}^{\cos }}{\sum_{i} B_{i i}^{\cos }}=\frac{\sum_{k \neq i} b_{i k} \cos \left(\theta_{i}-\theta_{k}\right)}{\sum_{i} \sum_{k \neq i} b_{i k} \cos \left(\theta_{i}-\theta_{k}\right)} \\
& =\frac{\sum_{j=1, j \neq i}^{N} B_{i j} \cos \left(\theta_{i}-\theta_{j}\right)}{\sum_{i} \sum_{j=1, j \neq i}^{N} B_{i j} \cos \left(\theta_{i}-\theta_{j}\right)}
\end{aligned}
$$

This modified susceptance matrix does not have an exact electrical interpretation. However, an approximate interpretation is:

$C_{i}^{D}\left(B^{\cos }\right)=\frac{\text { Net reactive power injected at bus } i \text { (quasi-ideal case) }}{\text { Total net reactive power injected in the network (quasi-ideal case) }}$ 


\subsection{Power traffic degree centrality}

The power traffic pseudo-Laplacian matrix $\tilde{L}_{i j}^{P T}$ defined by Equation (59) and power traffic degree matrix $\tilde{D}_{i j}^{P T}$ defined by Equation (58) can be used to specify a notion of the centrality. The power values are obtained using the Newton-Raphson load flow solution of a nonlinear system. Alternatively, the angles can be approximated by solving Equation (39) and the approximate power flow traffic can be obtained using Equation (22).

In this case, the power traffic degree matrix $\tilde{D}_{i j}^{P T}$ can be used to obtain the power traffic degree centrality $C_{i}^{D}(P T)$, which is given by:

$$
\begin{aligned}
C_{i}^{D}(P T) & =\frac{\tilde{D}_{i i}^{P T}}{\sum_{i i} \tilde{D}_{i i}^{P T}}=\frac{\sum_{j}\left|P_{i j}^{F}\right|}{\sum_{i} \sum_{j}\left|P_{i j}^{F}\right|} \\
& =\frac{\text { Total power traffic going through bus } i}{\text { Total power traffic in the network }}
\end{aligned}
$$

\subsection{Power loss degree centrality}

The power loss degree centrality is based on the power loss Laplacian matrix defined by Equation (62). The pseudo-adjacency matrix is given by $W_{i j}$ as defined in Equation (60) and is calculated using the actual real power loss in the network (or approximately as discussed above). The corresponding pseudo-degree matrix satisfies $\tilde{D}_{i j}^{W}=\tilde{A}_{i j}^{W}+\tilde{L}_{i j}^{W}$ and is computed using Equation (61). Note that:

$$
\begin{aligned}
\tilde{D}_{i i}^{W} & =\sum_{k \neq i} W_{i k}=\sum_{k \neq i} \frac{1}{2}\left(P_{i k}+P_{k i}\right) \\
& =\text { Total real power loss on all series lines connected to bus } i
\end{aligned}
$$

Then, the power loss degree centrality $C_{i}^{D}\left(P F^{W}\right)$ is computed as:

$$
\begin{aligned}
C_{i}^{D}\left(P F^{W}\right) & =\frac{\tilde{D}_{i i}^{W}}{\sum_{i} \tilde{D}_{i i}^{W}}=\frac{\sum_{k \neq i} \frac{1}{2}\left(P_{i k}+P_{k i}\right)}{\sum_{i} \sum_{k \neq i} \frac{1}{2}\left(P_{i k}+P_{k i}\right)} \\
& =\frac{\text { Total real power loss on all series lines connected to bus } i}{\text { Total real power loss in the network }}
\end{aligned}
$$

The electrical interpretation of power loss degree centrality is clear from Equation (69). 


\section{Brownout and blackout prediction}

This section presents the results of applying the new centrality measures to several networks, including the IEEE 30-bus, IEEE 57-bus and IEEE 300bus networks [36] as well as the WSCC 4941-bus real-world power grid [41]. The New York Independent System Operator (NYISO) network was also analyzed, but the results are not presented due to space constraints. The centrality distributions enabled the identification of nodes and branches in the networks that are important with respect to system vulnerability. A custom MATLAB [30] toolbox was used to compute the centrality measures and evaluate their ability to predict smart grid network brownouts and blackouts under random and targeted attacks [12].

\subsection{IEEE test networks}

This section analyzes the results obtained by applying the new centrality measures to the IEEE 30-bus, IEEE 57-bus and IEEE 300-bus networks.

Table 1 shows degree based centrality measures for the IEEE 30-bus network. All the centrality measure values were computed and plotted for the IEEE 30-bus, IEEE 57-bus and IEEE 300-bus networks. The topological centrality and susceptance based centrality (B) are important for structural vulnerability analyses. The modified susceptance based (Bcos), power traffic (PT) and power loss (PL) centralities are important for functional vulnerability analyses. The power traffic degree centrality $(\mathrm{PT})$ describes the real functional dynamics of a network.

The values in Table 1 reveal that the degree based centrality values are quite different, in general, with topological centrality having the most uniform values. Also, power loss centrality has negligible values for most buses. Moreover, there is agreement that the most central buses are the most prominent.

\subsection{Random attacks}

Monte Carlo simulations were used to study the effects of removing buses randomly $[29,43]$ and statistics were collected pertaining to power grid performance. In particular, the removal of specific combinations of buses and/or generators that caused blackouts were identified; otherwise, the extents of the corresponding brownouts were measured. Also the numbers of islands created in the networks were examined and whether or not the islands could satisfy 
Table 1: Degree based centrality measures for the IEEE 30-bus network.

\begin{tabular}{ccccccc}
\hline Node & Degree & $\begin{array}{c}\text { Topological } \\
\text { Centrality }\end{array}$ & $\begin{array}{c}\text { B } \\
\text { Centrality }\end{array}$ & $\begin{array}{c}\text { Bcos } \\
\text { Centrality }\end{array}$ & $\begin{array}{c}\text { PT } \\
\text { Centrality }\end{array}$ & $\begin{array}{c}\text { PL } \\
\text { Centrality }\end{array}$ \\
\hline 1 & 2 & 0.0244 & 0.0345 & 0.0204 & 0.1514 & 0.2883 \\
2 & 4 & 0.0488 & 0.0499 & 0.0426 & 0.3131 & 0.5234 \\
3 & 2 & 0.0244 & 0.0474 & 0.0384 & 0.0708 & 0.0698 \\
4 & 4 & 0.0488 & 0.0903 & 0.1392 & 0.1670 & 0.0269 \\
5 & 2 & 0.0244 & 0.0198 & 0.0067 & 0.0310 & 0.0450 \\
6 & 7 & 0.0854 & 0.1347 & 0.3099 & 0.2044 & 0.0417 \\
7 & 2 & 0.0244 & 0.0300 & 0.0154 & 0.0107 & 0.0017 \\
8 & 2 & 0.0244 & 0.0432 & 0.0318 & 0.0032 & 0.0001 \\
9 & 3 & 0.0366 & 0.0304 & 0.0158 & 0.0095 & 0.0000 \\
10 & 6 & 0.0732 & 0.0676 & 0.0780 & 0.0148 & 0.0002 \\
11 & 1 & 0.0122 & 0.0078 & 0.0010 & 0.0000 & 0.0000 \\
12 & 5 & 0.0610 & 0.0397 & 0.0269 & 0.0131 & 0.0011 \\
13 & 1 & 0.0122 & 0.0116 & 0.0023 & 0.0000 & 0.0000 \\
14 & 2 & 0.0244 & 0.0088 & 0.0013 & 0.0001 & 0.0000 \\
15 & 4 & 0.0488 & 0.0261 & 0.0116 & 0.0008 & 0.0008 \\
16 & 2 & 0.0244 & 0.0138 & 0.0036 & 0.0001 & 0.0001 \\
17 & 2 & 0.0244 & 0.0239 & 0.0104 & 0.0001 & 0.0000 \\
18 & 2 & 0.0244 & 0.0161 & 0.0044 & 0.0001 & 0.0000 \\
19 & 2 & 0.0244 & 0.0293 & 0.0146 & 0.0001 & 0.0000 \\
20 & 2 & 0.0244 & 0.0256 & 0.0112 & 0.0005 & 0.0000 \\
21 & 2 & 0.0244 & 0.0734 & 0.0918 & 0.0004 & 0.0001 \\
22 & 3 & 0.0366 & 0.0152 & 0.0853 & 0.0002 & 0.0000 \\
23 & 2 & 0.0244 & 0.0669 & 0.0022 & 0.0000 & 0.0000 \\
24 & 3 & 0.0366 & 0.0152 & 0.0038 & 0.0000 & 0.0000 \\
25 & 3 & 0.0366 & 0.0128 & 0.0028 & 0.0001 & 0.0000 \\
26 & 1 & 0.0122 & 0.0030 & 0.0001 & 0.0000 & 0.0000 \\
27 & 4 & 0.0488 & 0.0154 & 0.0040 & 0.0034 & 0.0004 \\
28 & 3 & 0.0366 & 0.0368 & 0.0233 & 0.0046 & 0.0000 \\
29 & 2 & 0.0244 & 0.0059 & 0.0006 & 0.0002 & 0.0001 \\
30 & 2 & 0.0244 & 0.0049 & 0.0004 & 0.0002 & 0.0002 \\
\hline & & & & & &
\end{tabular}

their loads. Every subsequent island was then checked for survivability or a blackout situation.

In every Monte Carlo simulation, the power traffic degree based centrality scores for the buses that created brownouts or blackouts were recorded and summed. The total percentage of the unsatisfied load versus the total centrality score for all the removed buses was plotted for each simulation scenario. Newton-Raphson power flow analysis was employed with a maximum of 50 iterations. Table 2 presents the parameters for each of the 13 simulation scenarios under random attacks. The vulnerability profiles of some of the IEEE test networks are presented and discussed below.

Figure 3 shows the variation in the total unsatisfied load as a function 
Table 2: Parameters for Monte Carlo simulations under random attacks (Cases 1-13).

\begin{tabular}{cccccc}
\hline Case & $\begin{array}{c}\text { IEEE } \\
\text { Model }\end{array}$ & $\begin{array}{c}\text { Power Traffic } \\
\text { Centrality Type }\end{array}$ & $\begin{array}{c}\text { No. Buses } \\
\text { Removed }\end{array}$ & $\begin{array}{c}\text { Generator Buses } \\
\text { Removed }\end{array}$ & $\begin{array}{c}\text { No. } \\
\text { Simulations }\end{array}$ \\
\hline 1 & 30 & Degree & 8 & Y & 20 \\
2 & 30 & Degree & 10 & N & 200 \\
3 & 30 & Degree & 10 & Y & 200 \\
4 & 57 & Degree & 20 & N & 20 \\
5 & 57 & Degree & 20 & Y & 20 \\
6 & 57 & Degree & 20 & N & 50 \\
7 & 57 & Degree & 20 & Y & 50 \\
8 & 57 & Degree & 20 & N & 100 \\
9 & 57 & Degree & 20 & Y & 100 \\
10 & 300 & Degree & 45 & N & 20 \\
11 & 300 & Degree & 45 & Y & 20 \\
12 & 300 & Degree & 35 & N & 100 \\
13 & 300 & Degree & 35 & Y & 100 \\
\hline
\end{tabular}

Figure 3: Case 1: IEEE 30-bus vulnerability profile when removing generator buses (20 simulations).

of the total score of the removed buses, where the scores were computed based on power traffic degree centrality. When all the loads are unsatisfied, the ratio is equal to one and the network is in a blackout situation. Note that no blackout occurs for a total centrality score less than 0.45. Moreover, whenever the total centrality score exceeds 0.5399 , a blackout is unavoidable. A least-squares fit of the data was used to create a linear model before the onset of a blackout. Up to eight buses were removed in the Monte Carlo simulations shown in Figure 3; the buses removed included generator buses.

Figure 4 shows the vulnerability of the IEEE 30-bus network when no generator buses were removed. Up to ten buses were removed in the Monte Carlo simulations (200 scenarios in all). Since generator buses were not removed, the onset of blackout was postponed until a total centrality score of 0.7237 was reached. Before the onset of a blackout, the ratio of total unsatisfied load increased as a linear function of the total centrality score of the removed buses. This is illustrated by the least-squares plot shown in Figure 4. The dependence is expected, but the standard deviation of the 
Figure 4: Case 2: IEEE 30-bus vulnerability profile without removing generator buses (200 simulations).

Figure 5: Case 3: IEEE 30-bus vulnerability profile when removing generator buses (200 simulations).

residual is high.

Figure 5 shows the vulnerability of the IEEE 30-bus network when up to ten buses were removed randomly (200 simulations were performed instead of 20 simulations in Case 1). The onset of blackout starts at a centrality score of 0.5502 . The transition to blackout occurs from the brownout stage between the centrality scores 0.4513 and 0.5502 .

Next, the proposed methodology was applied to the larger IEEE 57-bus network. The results were practically the same. In particular:

1. Removing generators increases power grid vulnerability. For example, the onset of blackout is reduced from 0.4168 in Case 4 (Figure 6) to 0.2963 in Case 5 (Figure 7).

2. The total unsatisfied load appears to be linearly dependent on the total centrality score of the removed buses. This can be seen by comparing the plots for Case 4 (Figure 6) and Case 5 (Figure 7).

3. When no generator buses are removed, the standard deviation of the residual is increased. This can be seen by comparing the plots for Case 4 (Figure 6) and Case 5 (Figure 7).

Up to 20 buses, including generator buses, were removed in Case 5, and 20 simulations were performed. The results are shown in Figure 7 . When all the generator buses were retained, the vulnerability profile was scattered due to the availability of excess generation. Only when the ability of the network to transmit excess generation is affected does the network go to a blackout situation.

\subsection{Statistical analysis of random attacks}

For each case listed in Table 2 (Cases 1 through 13), several parameters were computed and a goodness-of-fit $(\mathrm{GF})$ test was performed on the linear model of the vulnerability profile. The parameters included the maximum noblackout centrality point (CNB Max), minimum ensured blackout centrality point (CB Min) and the standard deviation of the residual at the pre-blackout 
Figure 6: Case 4: IEEE 57-bus vulnerability profile without removing generator buses.

Figure 7: Case 5: IEEE 57-bus vulnerability profile; removing generator buses increases the vulnerability.

point (STD Res). Additionally, the norm of the residual (Norm Res (GF)) and Pearson correlation coefficient (Pearson CC (GF)) were calculated. The results are shown in Table 3. Also, it was observed that 200 Monte Carlo simulations were adequate to obtain consistent results.

The norm of the residual is used to check the goodness-of-fit of data points. A general characteristic for an "acceptable" residual is that the value of the norm of the residual should be centered around zero. The Pearson correlation coefficient is a value between -1 and 1 ; the closer the coefficient is to either -1 or 1 , the stronger is the correlation between the variables [17, 35]. The results in Table 3 reveal that most of the data points pass the goodnessof-fit test. Thus, the predictions of brownouts and blackouts are statistically significant.

It is also important to note that, in a large network, damage concentrated at highly central buses is worse than damage that is diffused throughout the network. This observation is based on the maximum number of buses that can be removed.

\subsection{Analysis of targeted (centrality based) attacks}

In order to simulate targeted attacks by a malicious actor, buses with the highest centrality scores were removed (from the highest to the lower scores) either one bus at a time, two buses at a time or three buses at a time. The vulnerability profiles of the IEEE networks under these centrality based attacks are shown in Figure 8 (IEEE 30-bus network), Figure 9 (IEEE 57-bus network) and Figure 10 (IEEE 300-bus network).

For Cases 14, 15 and 16, removing the highest centrality score buses ensured blackout situations. As less central buses were removed, the networks went into brownout. Note that removing any one of the 100 most-central buses guarantees a blackout. Removing three buses at a time makes the situation worse.

\subsection{Vulnerability analysis of real power grids}

Experiments were also conducted with the WSCC real-world power grid with 4,941 nodes and 6,594 links [41] and the New York Independent System 
Table 3: Statistical results for random attacks on the IEEE test networks.

\begin{tabular}{ccccccccccc}
\hline Case & $\begin{array}{c}\text { IEEE } \\
\text { Model }\end{array}$ & $\begin{array}{c}\text { Power } \\
\text { Traffic } \\
\text { Centrality }\end{array}$ & $\begin{array}{c}\text { No. Buses } \\
\text { Removed }\end{array}$ & $\begin{array}{c}\text { Generation } \\
\text { Buses } \\
\text { Removed }\end{array}$ & $\begin{array}{c}\text { No. } \\
\text { Simulations }\end{array}$ & $\begin{array}{c}\text { CNB } \\
\text { Max }\end{array}$ & $\begin{array}{c}\text { CB } \\
\text { Min }\end{array}$ & $\begin{array}{c}\text { STD } \\
\text { Res }\end{array}$ & $\begin{array}{c}\text { Norm } \\
\text { Res } \\
\text { (GF) }\end{array}$ & $\begin{array}{c}\text { Pearson } \\
\text { CC } \\
\text { (GF) }\end{array}$ \\
\hline 1 & 30 & Degree & 8 & Y & 20 & 0.3504 & 0.5399 & 0.111 & 0.4353 \\
2 & 30 & Degree & 10 & N & 200 & 0.7149 & 0.7237 & 0.1888 & 1.4516 & 0.2693 \\
3 & 30 & Degree & 10 & Y & 200 & 0.4513 & 0.5502 & 0.1232 & 0.4946 & 0.1018 \\
4 & 57 & Degree & 20 & N & 20 & 0.4168 & 0.5202 & 0.1324 & 0.4669 & 0.6257 \\
5 & 57 & Degree & 20 & Y & 20 & 0.2963 & 0.4602 & 0.0964 & 0.0710 & 0.2458 \\
6 & 57 & Degree & 20 & N & 50 & 0.7739 & 0.8023 & 0.1845 & 0.6769 & 0.6102 \\
7 & 57 & Degree & 20 & Y & 50 & 0.2756 & 0.4502 & 0.0831 & 0.1062 & 0.2557 \\
8 & 57 & Degree & 20 & N & 100 & 0.5940 & 0.6102 & 0.1498 & 1.0043 & 0.3555 \\
9 & 57 & Degree & 20 & Y & 100 & 0.3230 & 0.4802 & 0.0874 & 0.1311 & 0.3160 \\
10 & 300 & Degree & 45 & N & 20 & 0.1856 & 0.4612 & 0.0573 & 0.0749 & 0.3308 \\
11 & 300 & Degree & 45 & Y & 20 & 0.1690 & 0.4580 & 0.0434 & 0.0675 & 0.1971 \\
12 & 300 & Degree & 35 & N & 100 & 0.2880 & 0.4566 & 0.0618 & 0.1415 & 0.1205 \\
13 & 300 & Degree & 35 & $\mathrm{Y}$ & 100 & 0.2730 & 0.4512 & 0.0631 & 0.0942 & 0.5128 \\
\hline
\end{tabular}

Figure 8: Case 14: IEEE 30-bus vulnerability profile under power traffic degree based centrality attacks (most central buses removed first).

Operator (NYISO) network with 2,935 nodes and 6,567 edges. In particular, experiments were conducted to evaluate the performance of the proposed modified centrality based approach in identifying vulnerabilities in real power grids. In the experiments, network dynamics was considered in terms of power flow distribution and network damage was assessed in terms of load shedding (i.e., total unsatisfied load).

Table 4 shows the analysis parameters used for the two networks. Note that, while the New York Independent System Operator (NYISO) network was analyzed, its results are not presented in this paper due to space constraints.

Figure 11 shows the performance of the WSCC power grid under centrality based (targeted) attacks. The results clearly demonstrate that targeted attacks are very effective at fragmenting a network because buses with the largest centrality degrees or largest power flows are the first targets of attack and removing even a small fraction of buses can have dramatic negative consequences. When targeted attacks are executed, only a small fraction of buses need to be eliminated to disrupt the network or to cause a brownout. The approach gives good estimates of the thresholds for disrupted networks under targeted attacks; these thresholds can be compared with the thresholds obtained from real power grid data. The results of the analysis can be used to determine brownout and blackout states. This provides a deep un- 
Figure 9: Case 15: IEEE 57-bus vulnerability profile under power traffic degree based centrality attacks (most central buses removed first).

Figure 10: Case 16: IEEE 300-bus vulnerability profile under power traffic degree based centrality attacks (most central buses removed first).

derstanding of the intrinsic robustness of smart power grids that can be used to develop new communications architectures and control schemes to compensate for intrinsic network vulnerabilities and enhance survivability and robustness.

The centrality measures described in this paper can be extended to compute subnetwork centrality scores for interconnected subnetworks. Figure 12 shows three interconnected subnetworks joined at Node 8 . For each network, all the degree based centrality measures may be computed, including the subnetwork centrality values. Under random and targeted attacks, the subnetworks provide the same results as when the network is considered as a whole. Note that network vulnerability is assessed in terms of power flow centrality or power traffic centrality. Although the topologies of the subnetworks may not be known, the power flow or power traffic centrality values can help estimate the possible failures and vulnerabilities in the subnetworks. The results obtained for a network based on power traffic centrality can be rerun to identify vulnerabilities in its subnetworks. Even if information about subnetwork topology is unavailable, the subnetwork capacity can be used to determine vulnerabilities using the power traffic and power loss based centrality measures. The approach derives from the facts that real networks have both structural and functional parameters, and that network structure and function cannot exist without each other.

\section{Conclusions}

The pseudo-Laplacian, pseudo-adjacency and pseudo-degree matrices presented in this paper are valuable analysis tools for smart grid networks. The matrices were developed after a careful analysis of the analogies between the admittance matrix and the Laplacian matrix describing network topology. A pseudo-diagonal degree matrix represents the sum of the susceptances connected to a bus (diagonal elements) and a pseudo-adjacency matrix represents the susceptance of the lines connecting two buses. These matrices 
Table 4: Analysis parameters for real-world power grid networks.

\begin{tabular}{lcc}
\hline Analysis Parameters & WSCC & NYISO \\
\hline Number of Nodes $(V)$ & 4,941 & 2,935 \\
Number of Links $(E)$ & 6,594 & 6,567 \\
Average Node Degree $(k)$ & 2.67 & 4.47 \\
Global Clustering Coeff. $\left(C C_{\text {global }}\right)$ & 0.0801 & 0.2134 \\
Average Path Length $(l)$ & 18.98 & 16.43 \\
Algebraic Connectivity $\left(\lambda_{2}(L)\right)$ & 0.0007 & 0.0014 \\
Pearson Correlation Coeff. $(\rho)$ & 0.0035 & 0.4593 \\
\hline
\end{tabular}

Figure 11: WSCC U.S. grid vulnerability profile under centrality based attacks (most central buses removed first).

were used to specify four new weighted graph matrices: (i) susceptance matrix; (ii) modified susceptance matrix; (iii) power traffic matrix; and (iv) power loss matrix. These four weighted graph matrices were then used to define four new degree centrality measures. Each degree centrality has its own characteristics and a distinct electrical interpretation.

The new weighted graph centrality measures and their electrical interpretations are important for identifying network vulnerabilities. Removing buses with a total centrality score greater than 0.45 leads to a blackout situation when the available generation and load are not balanced. When all the generator buses are retained, the network vulnerability profile is scattered due to the availability of excess generation. Only when the ability of the network to transmit excess generation is disrupted, does the network move to a blackout situation.

A total of 13 scenarios involving test power grids were analyzed using Monte Carlo simulation. Every simulation scenario involved removing a subset of buses and performing a complete nonlinear Newton-Raphson power flow analysis to compute the power traffic matrix and the corresponding centrality score. The Monte-Carlo simulations demonstrate conclusively that the centrality measures based on the power traffic matrix are reliable indicators of the total unsatisfied load ratio (load taken offline due to an attack divided by the total load demand). A key result is that when the total centrality score of the removed buses exceeds a threshold estimated via Monte Carlo 
Figure 12: Interconnected subnetwork centrality analysis.

simulation, a sudden and dramatic jump to a blackout situation is ensured.

Statistical analysis reveals that the majority of data points generated by the simulations pass goodness-of-fit tests. The linear models describing brownout situations also pass the goodness-of-fit tests. Thus, the prediction results for brownout and blackout situations are statistically significant. Furthermore, the analysis reveals that, in a large network, damage that is concentrated in a few highly central buses is worse than damage that is more diffused throughout the network.

The analyses of centrality based attacks in Cases 14, 15 and 16 reveal that removing buses with the highest centrality scores from top to bottom ensure blackout situations. However, as the removed buses become less central, the networks go into brownout situations. Such vulnerability analyses of real power grids under centrality based attacks can help determine the effectiveness of attacks and well as solutions for avoiding major grid failures.

Simulations of random and targeted (power traffic centrality based) attacks on test and real power grids can help determine the load variations and vulnerability profiles of the grids. The power traffic degree based centrality measures actually play an important role in analyzing vulnerability in terms of smart grid brownouts and blackouts; they also help describe the true dynamics of smart grid networks.

The methodology and results presented in this paper should be useful to the electric power sector and to critical infrastructure protection researchers for identifying vulnerabilities and predicting brownout and blackout situations, and developing mitigation solutions for current and future smart grid networks.

\section{Acknowledgements}

This research was supported by the U.S. Defense Threat Reduction Agency and Pennsylvania State University under Contract DTRA01-03-D-0010/0020 and Subcontract S03-34. The authors also acknowledge the support of North Carolina A\&T State University, Greensboro, North Carolina and Bharati Vidyapeeth Deemed University College of Engineering, Pune, India. 
IMPORTANT NOTE TO IJCIP TYPESETTERS: I have checked and edited the references in this paper myself. Please DO NOT MODIFY the references - except to add hyperlinks. Please contact the Journal Manager Ms. Ramya Vasudevan if you have any questions.

Professor Sujeet Shenoi, Editor-in-Chief, IJCIP

\section{References}

[1] M. Amin, National infrastructure as complex interactive networks, in Automation, Control and Complexity: An Integrated Approach, T. Samad and J. Weyrauch (Eds.), John Wiley and Sons, New York, pp. 263-286, 2000.

[2] M. Amin, Enabling a resilient and secure North American electric power grid, keynote address at the Fourth International Symposium on Resilient Control Systems, 2011.

[3] G. Andersson, Modeling and Analysis of Electric Power Systems: Power Flow Analysis, Fault Analysis, Power Systems Dynamics and Stability, Power Systems Laboratory, Swiss Federal Institute of Technology Zurich, Zurich, Switzerland, 2008.

[4] G. Andersson, P. Donalek, R. Farmer, N. Hatziargyriou, I. Kamwa, P. Kundur, N. Martins, J. Paserba, P. Pourbeik, J. Sanchez-Gasca, R. Schulz, A. Stankovic, C. Taylor and V. Vittal, Causes of the 2003 major grid blackouts in North America and Europe, and recommended means to improve system dynamic performance, IEEE Transactions on Power Systems, vol. 20(4), pp. 1922-1928, 2005.

[5] T. Biyikoglu, J. Leydold and P. Stadler, Laplacian Eigenvectors of Graphs: Perron-Frobenius and Faber-Krahn Type Theorems, SpringerVerlag, Berlin Heidelberg, Germany, 2007.

[6] E. Bompard, R. Napoli and F. Xue, Analysis of structural vulnerabilities in power transmission grids, International Journal of Critical Infrastructure Protection, vol. 2(1-2), pp. 5-12, 2009.

[7] S. Borgatti, Centrality and network flow, Social Networks, vol. 27(1), pp. 55-71, 2005. 
[8] S. Borgatti and M. Everett, A graph-theoretic perspective on centrality, Social Networks, vol. 28(4), pp. 466-484, 2006.

[9] A. Chakrabortty and M. Ilic (Eds.), Control and Optimization Methods for Electric Smart Grids, Springer-Verlag, New York, 2012.

[10] R. Chen, A. Cohn, N. Fan and A. Pinar, N-k- $\epsilon$ Survivable Power System Design, Cornell University Library, Cornell University, Ithaca, New York (arxiv.org/abs/1201.1530v1), 2012.

[11] R. Chen, A. Cohn, N. Fan and A. Pinar, Contingency-risk informed power system design, IEEE Transactions on Power Systems, vol. 29(5), pp. 2087-2096, 2014.

[12] P. Chopade, Robustness and Survivability of Smart Power Grid and SCADA Networks When Subjected to Severe Emergencies, Vulnerabilities and WMD Attacks, Ph.D. Dissertation, Department of Computational Science and Engineering, North Carolina A\&T State University, Greensboro, North Carolina, 2013.

[13] P. Crucitti, V. Latora, M. Marchiori and A. Rapisarda, Efficiency of scale-free networks: Error and attack tolerance, Physica A: Statistical Mechanics and its Applications, vol. 320, pp. 622-642, 2003.

[14] P. de Meoa, E. Ferrara, G. Fiumara and A. Ricciardello, A novel measure of edge centrality in social networks, Knowledge-Based Systems, vol. 30, pp. 136-150, 2012.

[15] N. Deo, Graph Theory with Applications to Engineering and Computer Science, Prentice-Hall of India, New Delhi, India, 2004.

[16] V. Donde, V. Lopez, B. Lesieutre, A. Pinar, C. Yang and J. Meza, Identification of severe multiple contingencies in electric power networks, Proceedings of the Thirty-Seventh Annual North American Power Symposium, pp. 59-66, 2005.

[17] S. Dowdy, S. Weardon and D. Chilko, Statistics for Research, John Wiley and Sons, Hoboken, New Jersey, 2004.

[18] M. Dunn and I. Wigert, Critical Information Infrastructure Protection, International CIIP Handbook 2004, Swiss Federal Institute of Technology Zurich, Zurich, Switzerland, 2004. 
[19] T. Feyessa and M. Bikdash, Geographically-sensitive network centrality and survivability assessment, Proceedings of the Forty-Third IEEE Southeastern Symposium on System Theory, pp. 18-23, 2011.

[20] L. Freeman, Centrality in social networks - Conceptual clarification, Social Networks, vol. 1(3), pp. 215-239, 1978/79.

[21] J. Grainger and W. Stevenson, Power System Analysis, McGraw-Hill, New York, 1994.

[22] L. Grigsby (Ed.), The Electric Power Engineering Handbook: Power Systems, CRC Press, Boca Raton, Florida, 2012.

[23] Y. Hase, Handbook of Power Systems Engineering, John Wiley and Sons, Chichester, United Kingdom, 2007.

[24] P. Hines and S. Blumsack, A centrality measure for electrical networks, Proceedings of the Forty-First Hawaii International Conference on System Sciences, 2008.

[25] S. Jin, Z. Huang, Y. Chen, D. Chavarria-Miranda, J. Feo and P. Wong, A novel application of parallel betweenness centrality to power grid contingency analysis, Proceedings of the IEEE International Symposium on Parallel and Distributed Processing, 2010.

[26] D. Koschutzki, K. Lehmann, L. Peeters, S. Richter, D. Tenfelde-Podehl and O. Zlotowski, Centrality indices, in Network Analysis: Methodological Foundations, U. Brandes and T. Erlebach (Eds.), Springer-Verlag, Berlin Heidelberg, Germany, pp. 16-61, 2005.

[27] P. Lancaster and M. Tismenetsky, The Theory of Matrices with Applications, Academic Press, San Diego, California, 1985.

[28] P. Marsden, Egocentric and sociocentric measures of network centrality, Social Networks, vol. 24(4), pp. 407-422, 2002.

[29] W. Martinez and A. Martinez, Computational Statistics Handbook with MATLAB, Chapman and Hall/CRC, Boca Raton, Florida, 2008.

[30] MathWorks, MATLAB Release 2015a products with license-related changes, Natick, Massachusetts, 2015. 
[31] R. Merris, Laplacian graph eigenvectors, Linear Algebra and its Applications, vol. 278(1-3), pp. 221-236, 1998.

[32] D. Newman, B. Carreras, V. Lynch and I. Dobson, Exploring complex systems aspects of blackout risk and mitigation, IEEE Transactions on Reliability, vol. 60(1), pp. 134-143, 2011.

[33] A. Pinar, J. Meza, V. Donde and B. Lesieutre, Optimization strategies for the vulnerability analysis of the electric power grid, SIAM Journal on Optimization, vol. 20(4), pp. 1786-1810, 2010.

[34] F. Saccomanno, Electric Power Systems: Analysis and Control, WileyIEEE Press, Piscataway, New Jersey, 2003.

[35] M. Samuels and J. Witmer, Statistics for the Life Sciences, Prentice Hall, Upper Saddle River, New Jersey, 2003.

[36] University of Washington - Department of Electrical Engineering, Power Systems Test Case Archive, Seattle, Washington (www.ee.washington. edu/research/pstca), 2015.

[37] A. von Meier, Electric Power System - A Conceptual Introduction, John Wiley and Sons, Hoboken, New Jersey, 2006.

[38] Q. Wang, J. Watson and Y. Guan, Two-stage robust optimization for N-k contingency-constrained unit commitment, IEEE Transactions on Power Systems, vol. 28(3), pp. 2366-2375, 2013.

[39] W. Wang and Z. Lu, Cyber security in the smart grid: Survey and challenges, Computer Networks, vol. 57(5), pp. 1344-1371, 2013.

[40] Z. Wang, A. Scaglione and R. Thomas, Electrical centrality measures for electric power grid vulnerability analysis, Proceedings of the Firty-Ninth IEEE Conference on Decision and Control, pp. 5792-5797, 2010.

[41] Western Electricity Coordinating Council (WECC), Transmission Expansion Planning Datasets, Salt Lake City, Utah (www.wecc.biz/ TransmissionExpansionPlanning/Pages/Datasets . aspx), 2015.

[42] E. Zio and R. Piccinelli, Randomized flow model and centrality measure for electrical power transmission network analysis, Reliability Engineering and System Safety, vol. 95(4), pp. 379-385, 2010. 
[43] E. Zio, L. Podofillini and V. Zille, A combination of Monte Carlo simulation and cellular automata for computing the availability of complex network systems, Reliability Engineering and System Safety, vol. 91(2), pp. 181-190, 2006. 


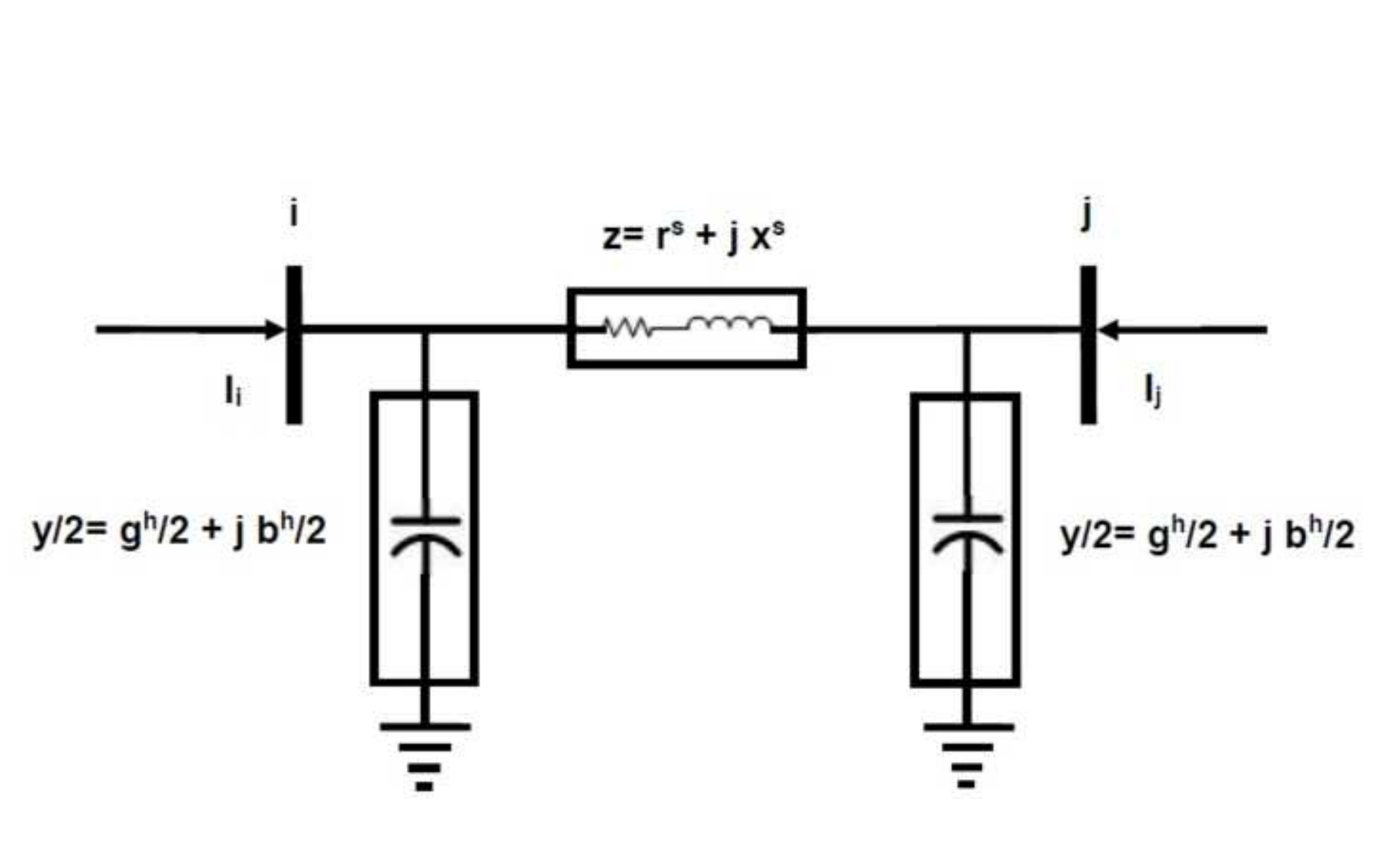

res
要

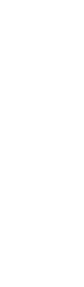




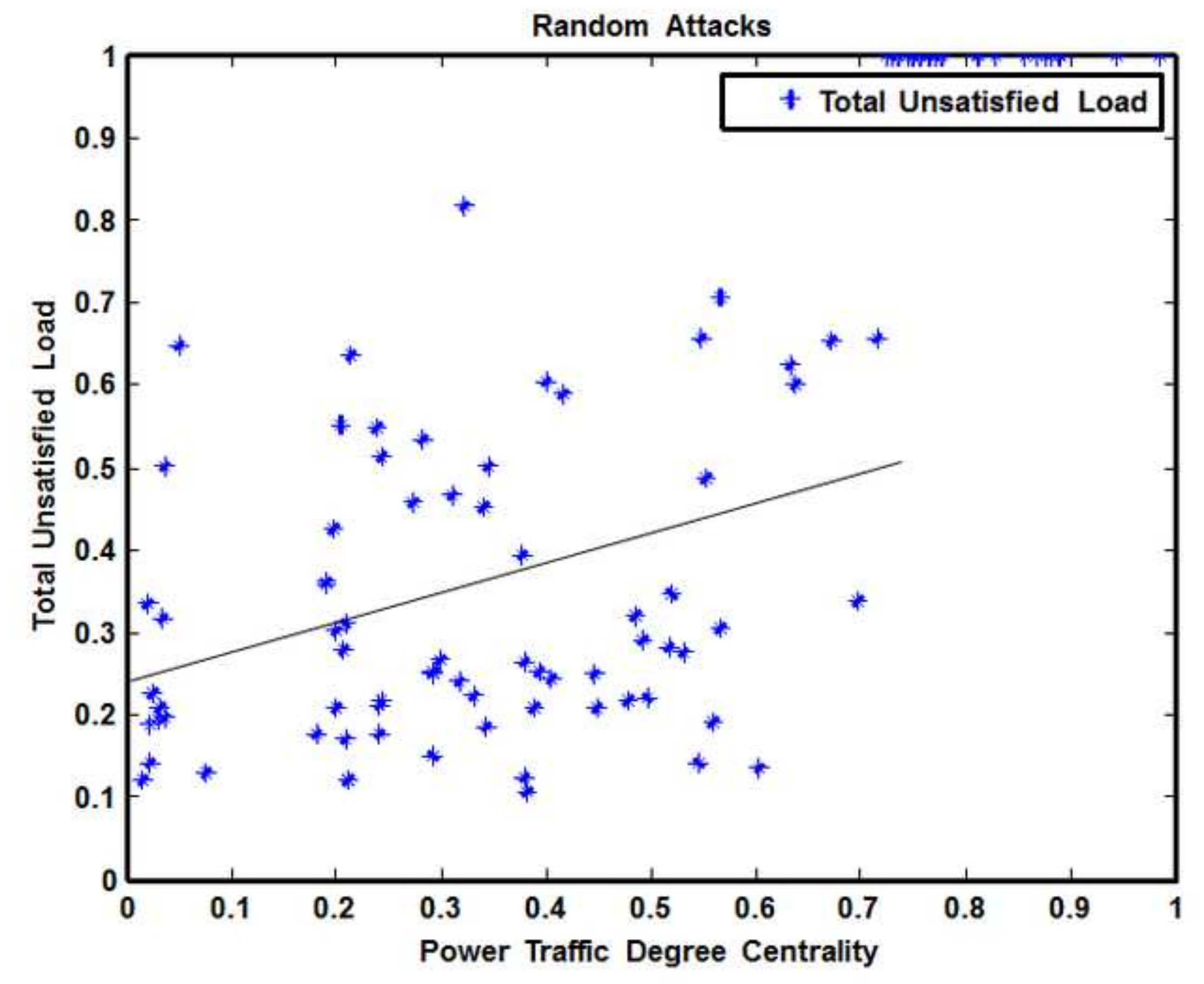




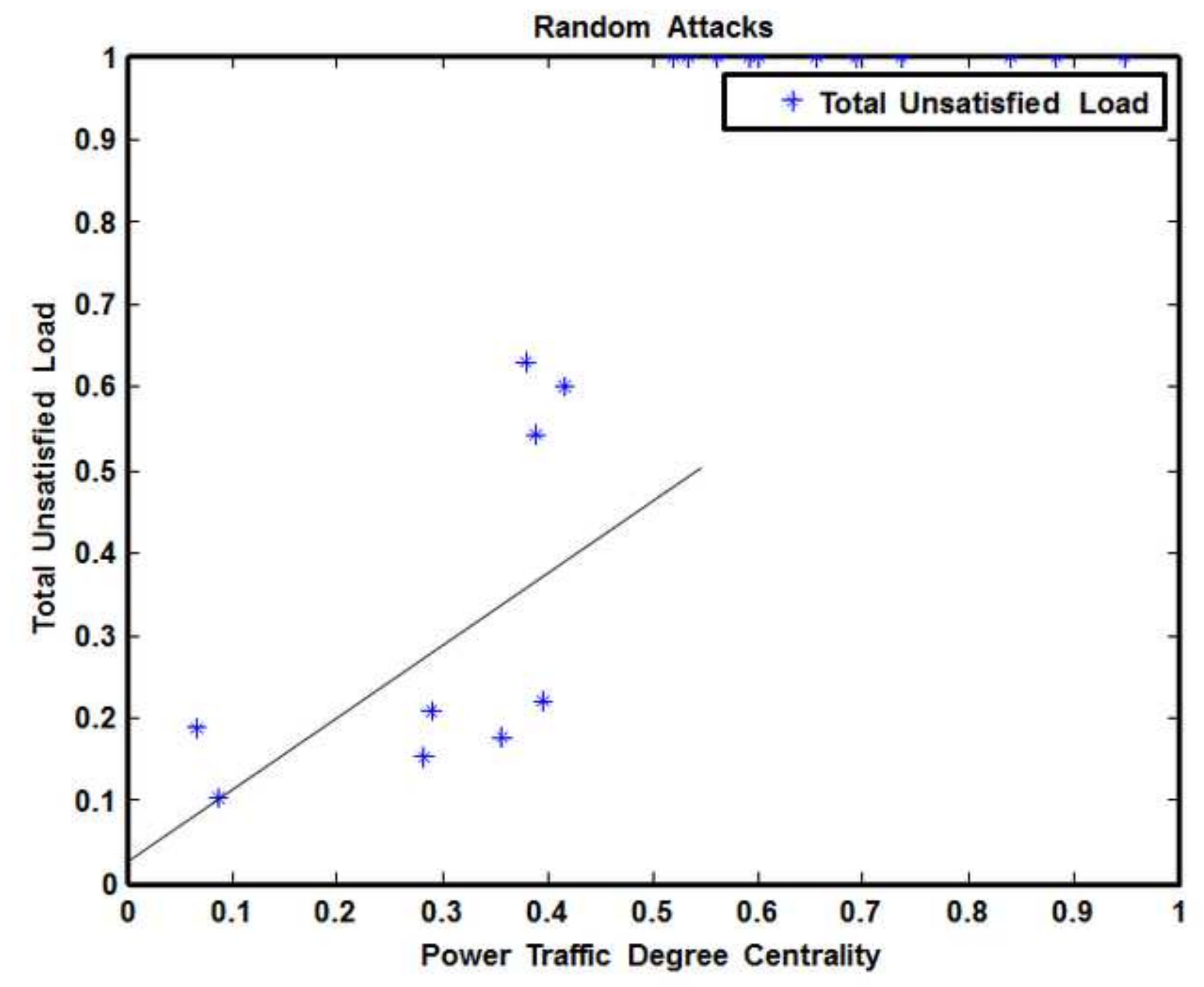




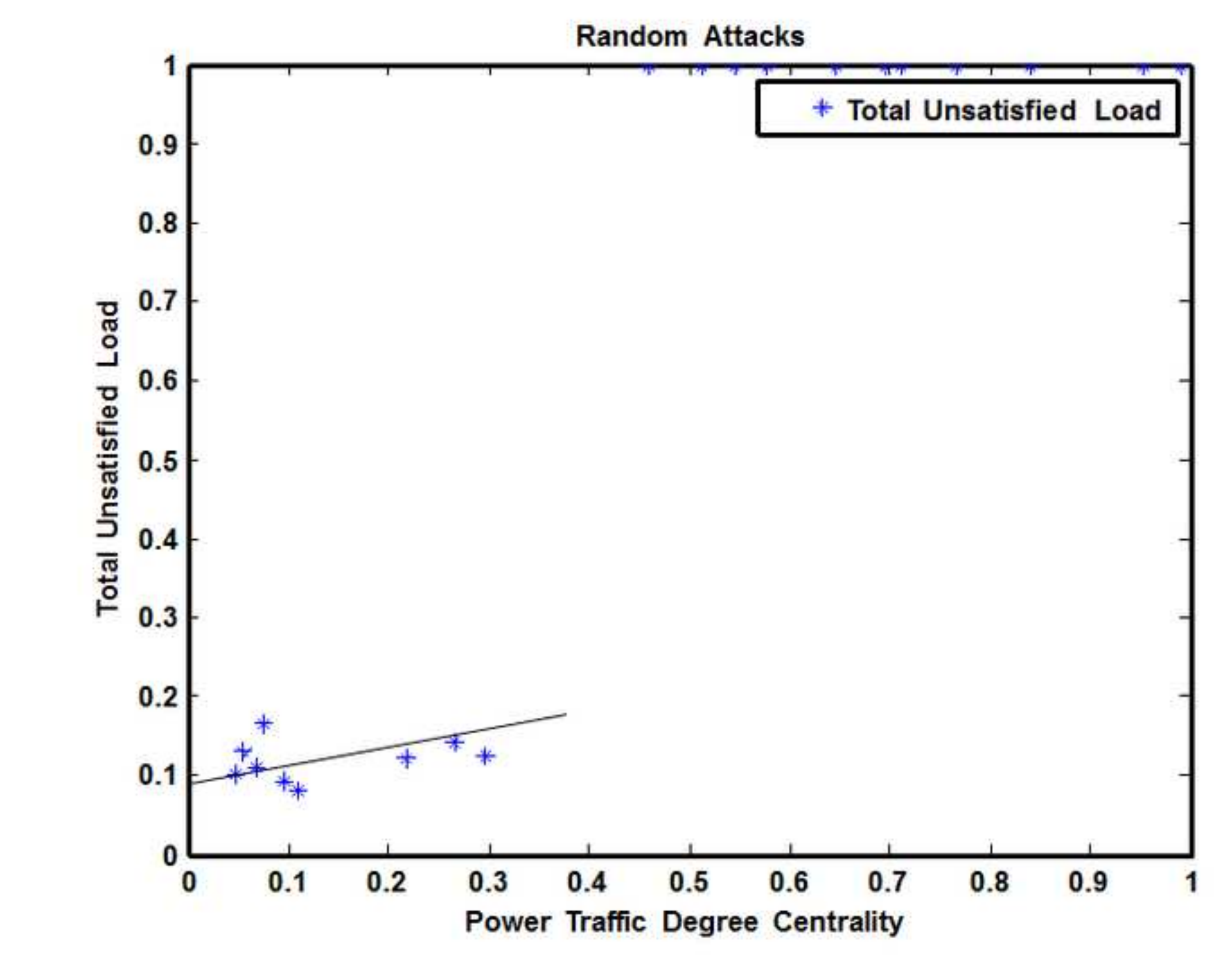

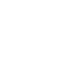

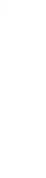

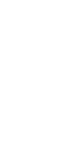
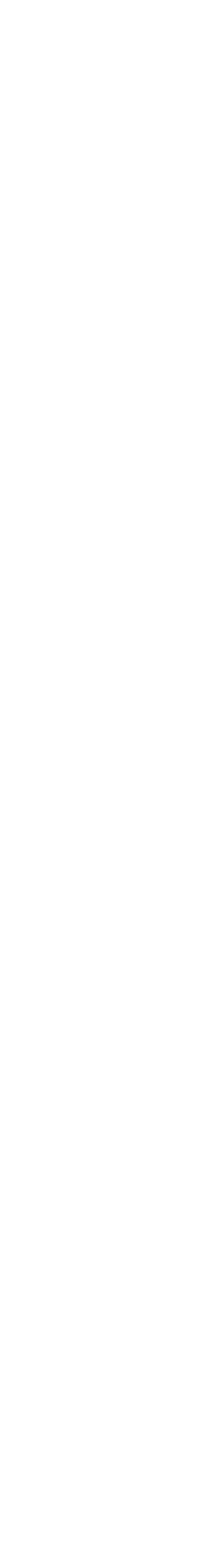


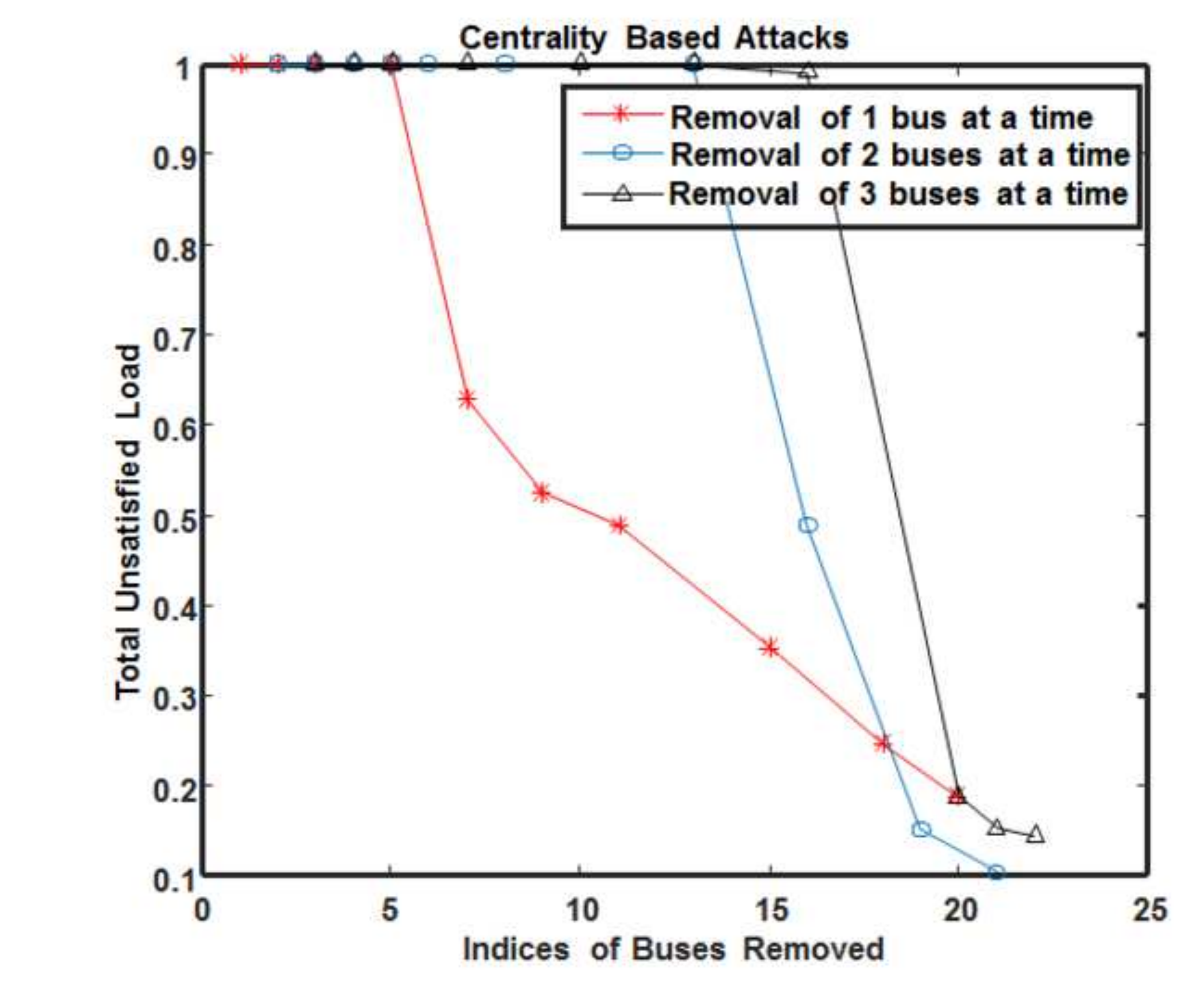

(

.

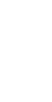

(-
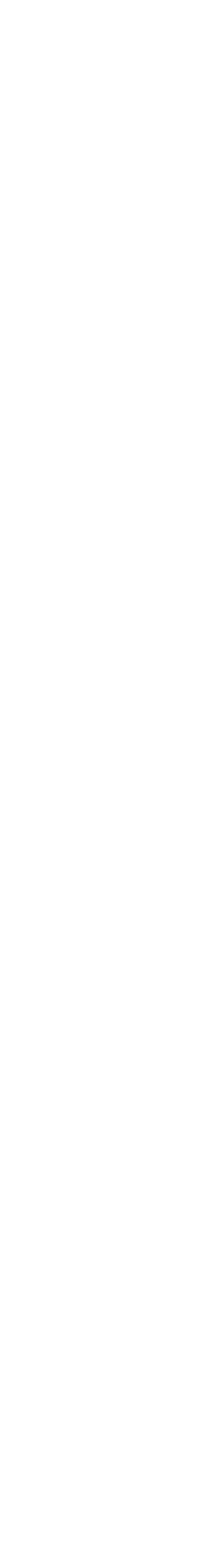


\section{Centrality Based Attacks}

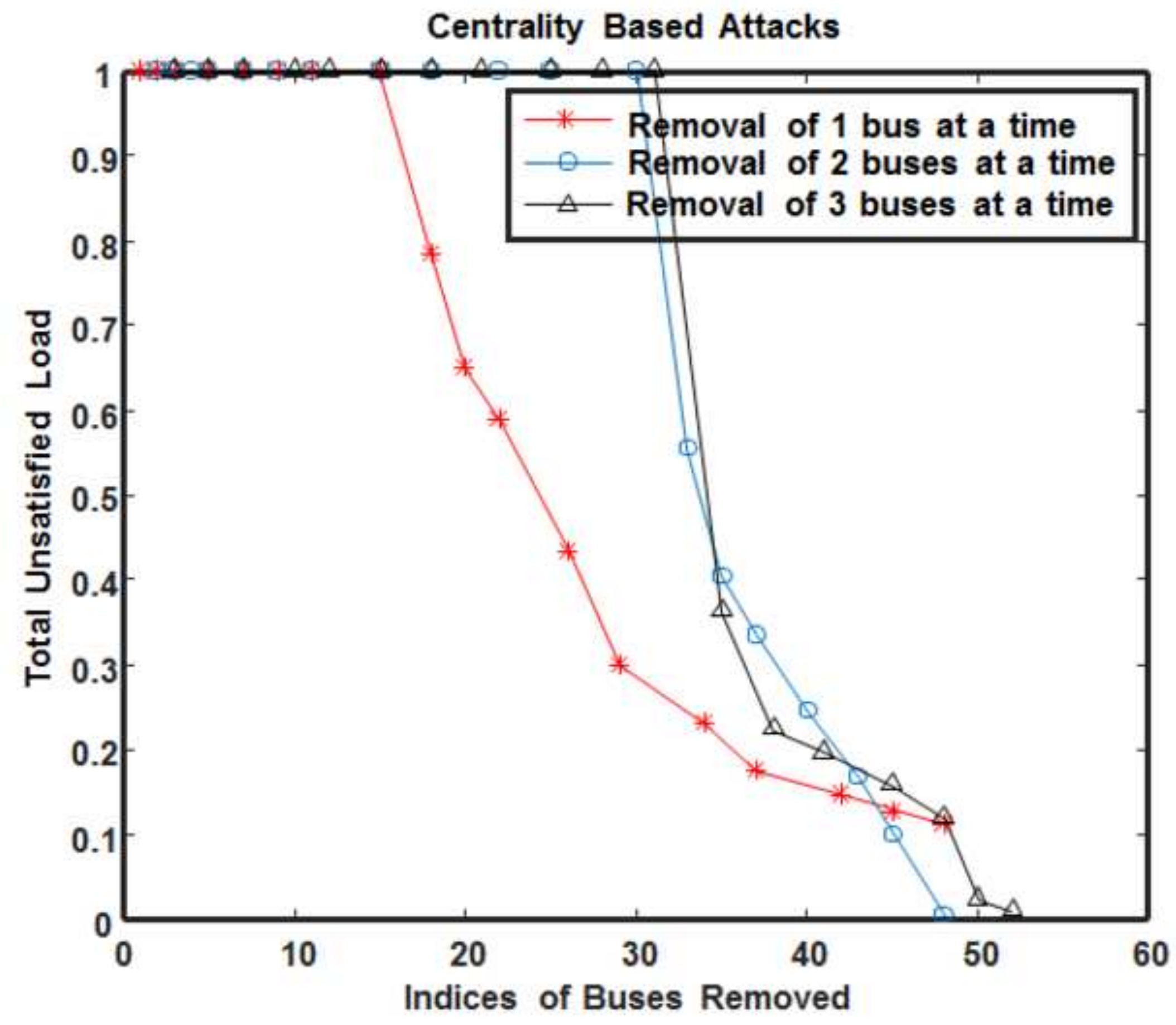




\section{Centrality Based Attacks}

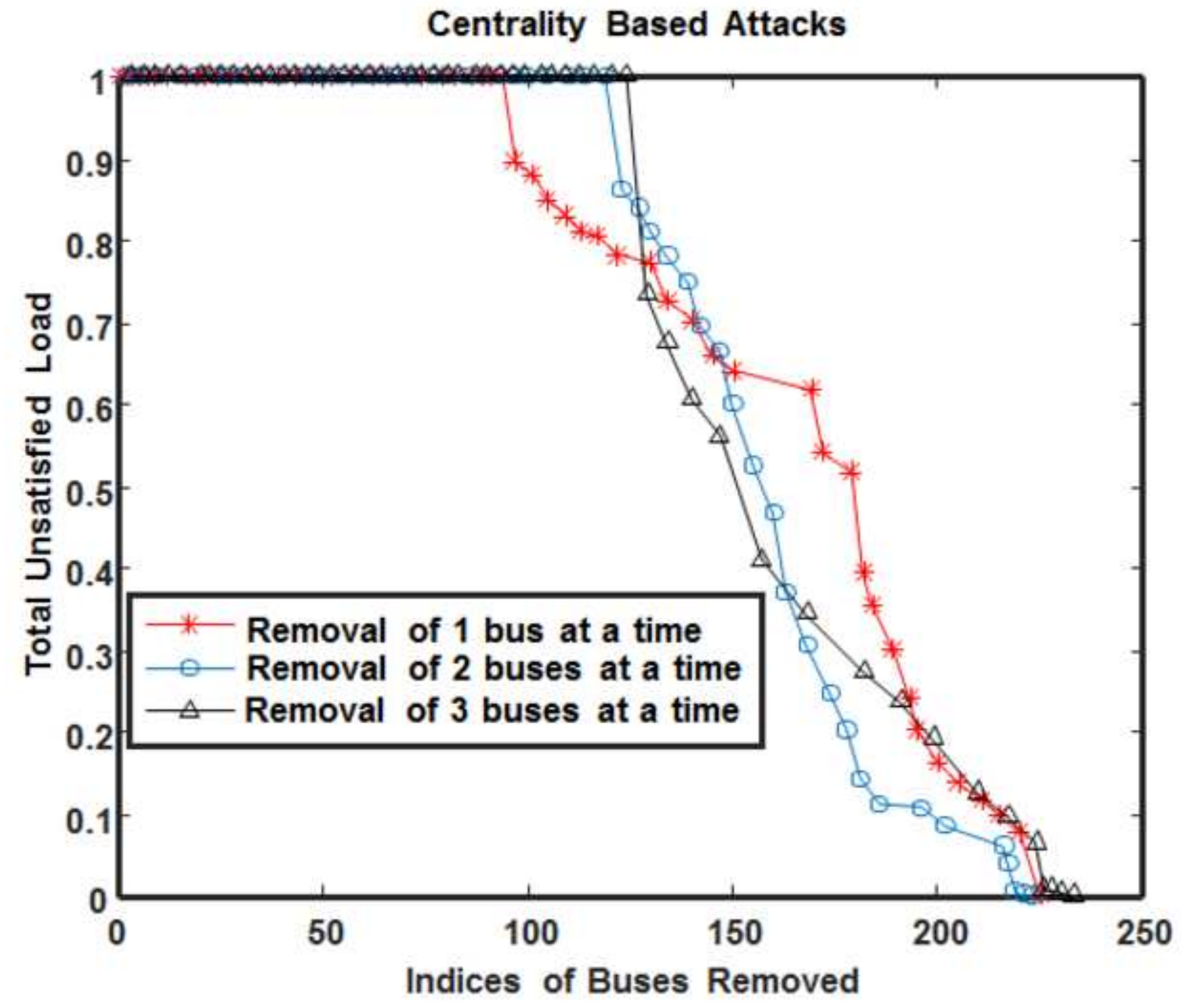




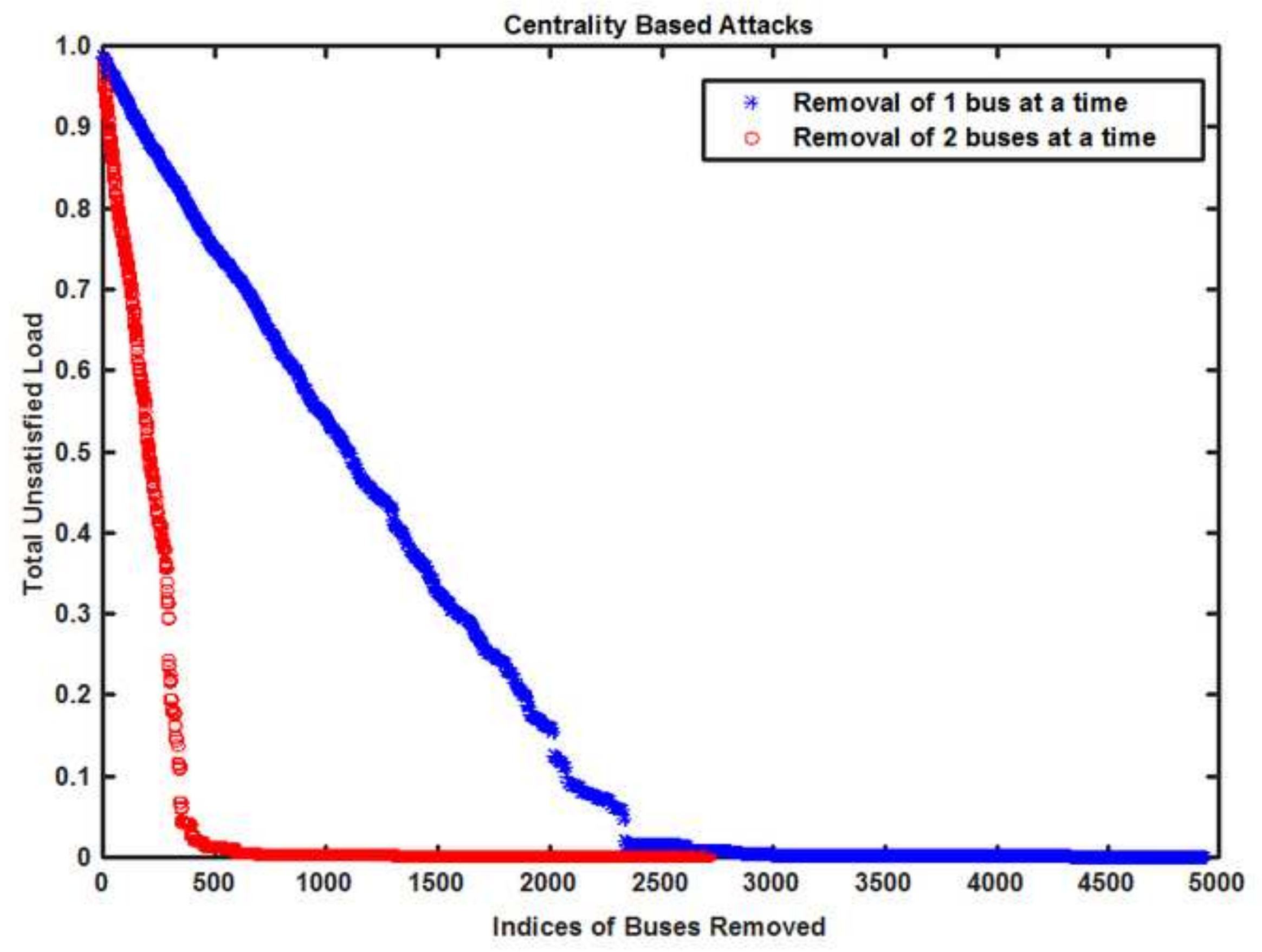




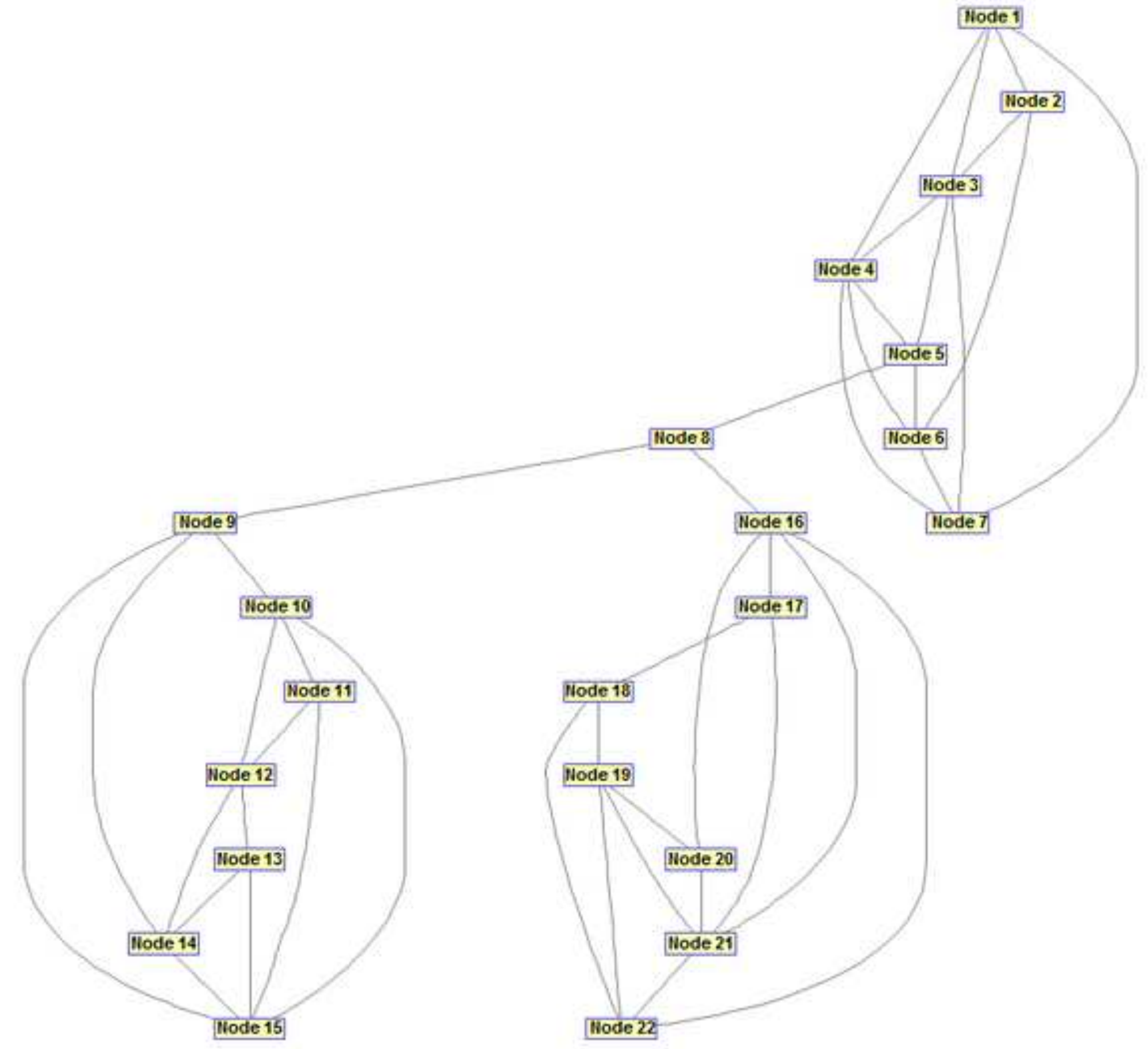

